\title{
Myeloid cell-targeted STAT3 inhibition sensitizes head and neck cancers to radiotherapy and T cell-mediated immunity
}

\author{
Dayson Moreira, ${ }^{1}$ Sagus Sampath, ${ }^{2}$ Haejung Won, ${ }^{1}$ Seok Voon White, ${ }^{1}$ Yu-Lin Su, ${ }^{1}$ Marice Alcantara, ${ }^{1}$ Chongkai Wang, ${ }^{1}$ Peter Lee, ${ }^{1}$ \\ Ellie Maghami, ${ }^{3}$ Erminia Massarelli, ${ }^{4}$ and Marcin Kortylewski ${ }^{1}$
}

${ }^{1}$ Department of Immuno-Oncology, Beckman Research Institute at City of Hope, Duarte, California, USA. ${ }^{2}$ Radiation Oncology, ${ }^{3}$ Surgery Department, and ${ }^{4}$ Medical Oncology and Experimental Therapeutics, City of Hope, Duarte, California, USA.

\begin{abstract}
The tumor microenvironment affects the outcome of radiotherapy against head and neck squamous cell carcinoma (HNSCC). We recently found that tolerogenic myeloid cells accumulate in the circulation of HNSCC patients undergoing radiotherapy. Here, we analyzed tumor-containing lymph node biopsies collected from these patients. After 2 weeks of radiotherapy, we found an increase in tumor-associated macrophages (TAMs) with activated STAT3, while CD8 ${ }^{+}$T cells were reduced as detected using multiplex IHC. Gene expression profiling indicated upregulation of M2 macrophage-related genes (CD163, CD206), immunosuppressive mediators (ARG1, LIF, TCFB1), and Th2 cytokines (IL4, IL5) in irradiated tumors. We next validated STAT3 as a potential target in human HNSCC-associated TAMs, using UM-SCC1 xenotransplants in humanized mice. Local injections of myeloid cell-targeted STAT3 antisense oligonucleotide (CpG-STAT3ASO) activated human DCs/macrophages and promoted $\mathrm{CD}^{+} \mathrm{T}$ cell recruitment, thereby arresting UM-SCC1 tumor growth. Furthermore, CpG-STAT3ASO synergized with tumor irradiation against syngeneic HPV+ $\mathrm{mEERL}$ and HPV- MOC2 HNSCC tumors in mice, triggering tumor regression and/or extending animal survival. The antitumor immune responses were $\mathrm{CD8} 8^{+}$and $\mathrm{CD4}{ }^{+} \mathrm{T}$ cell dependent and associated with the activation of antigen-presenting cells (DCs/M1 macrophages) and increased CD8 ${ }^{+}$ to regulatory $\mathrm{T}$ cell ratio. Our observations suggest that targeted inhibition of STAT3 in tumor-associated myeloid cells augments the efficacy of radiotherapy against HNSCC.
\end{abstract}

\section{Introduction}

Head and neck squamous cell carcinoma (HNSCC) is the sixth leading malignancy by incidence worldwide, with less than half of patients surviving for 5 years (1). HPV infection is one of the major risk factors for HNSCC. Approximately $80 \%$ of the newly diagnosed HNSCC cases represent the HPV ${ }^{-}$type, while the remaining $\mathrm{HPV}^{+} \mathrm{HNSCC}$ cases are pathologically and molecularly distinct malignancies with better responsiveness to chemotherapy and radiation therapy (RT) and improved patient survival $(1,2)$. These differences are partly related to immunogenicity of HPV-derived E6 and E7 oncoproteins, higher infiltration of $\mathrm{HPV}^{+} \mathrm{HNSCC}$ with $\mathrm{CD}^{+} \mathrm{T}$ lymphocytes, and better responsiveness to immune checkpoint blockade using monoclonal antibodies against programmed cell death-1 receptor (PD-1) compared

Author's note: DM and SS contributed equally to this work.

Conflict of interest: KK and DM are inventors on a patent application (US PTO $10,758,624)$ submitted by City of Hope that covers the design of oligonucleotides presented in this report. MK is a cofounder of iSTAT Therapeutics Inc. and a scientific advisor to Scopus Biopharma Inc., companies focused on oligonucleotide-based cancer immunotherapies.

Copyright: (c) 2021, American Society for Clinical Investigation.

Submitted: February 4, 2020; Accepted: November 18, 2020;

Published: January 19, 2021

Reference information: / Clin Invest. 2021;131(2):e137001.

https://doi.org/10.1172/JCl137001. with $\mathrm{HPV}^{-}$cancer patients (3-5). Despite the presence of the immune infiltrate, only a minority of HNSCC patients benefit from the emerging cancer immunotherapies, likely due to inhibitory effects of a potently immunosuppressive tumor microenvironment (TME) $(5,6)$. Current treatment of advanced HNSCC involves surgery and the combination of RT with concomitant chemotherapy or cetuximab, referred to as combined modality therapy (CMT) $(7,8)$. However, the survival rates have not markedly increased in recent decades (9) due to significant resistance of cancer cells and the TME to CMT (10-12).

The TME in head and neck tumors is characterized by the presence of proinflammatory mediators and immunosuppressive cells such as tumor-associated macrophages (TAMs) (13), myeloid-derived suppressor cells (MDSCs) $(14,15)$, and regulatory T cells (Tregs) $(5,6)$. The extrinsic effects of the TME are likely influencing the outcome of RT $(12,16,17)$. Although radiationinduced cell death can trigger immune activation and so-called abscopal effects, more often it results in tolerogenic signaling, tumor neovascularization, and cancer relapse. The components of dying cells, such as mitochondrial DNA, are recognized by innate immune receptors, such as Toll-like receptor 9 (TLR9) (18-20). Tolerogenic STAT3 activity in tumor-associated myeloid cells can alter the outcome of TLR9 signaling to promote low-grade inflammation with release of immunoregulatory and proangiogenic mediators $(21,22)$. We previously showed that TLR9 activation 
A

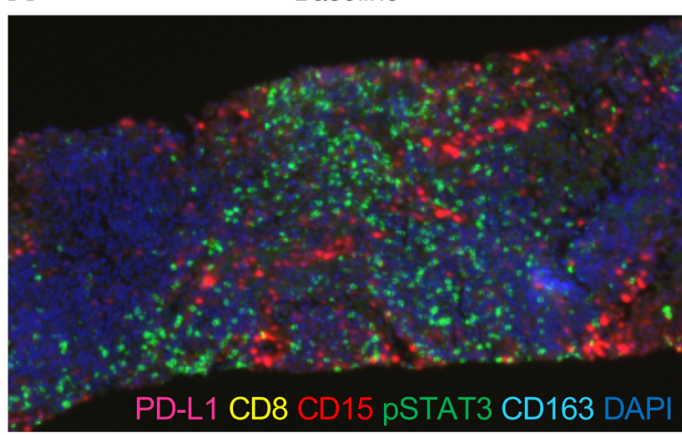

Week 2

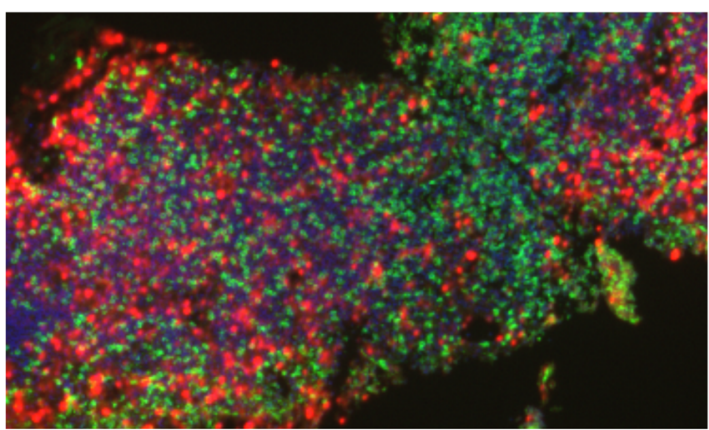

B

C

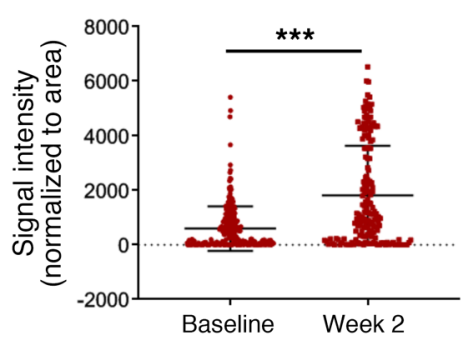

E

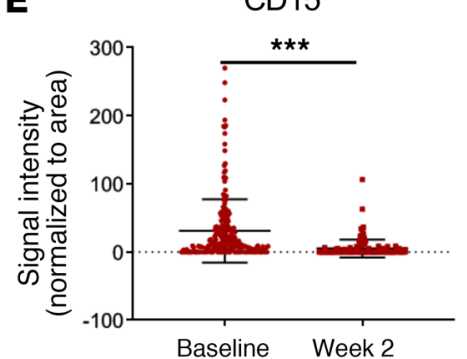

pSTAT3

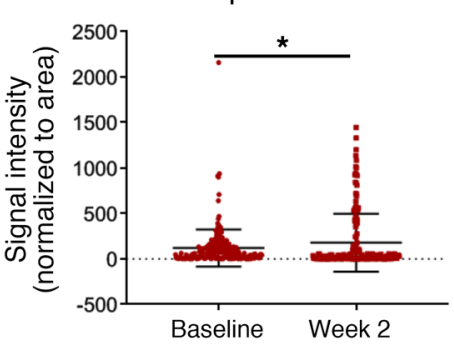

$\mathbf{F}$

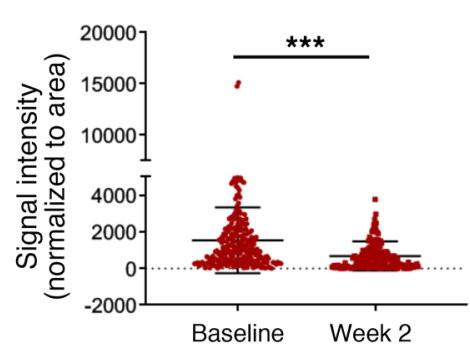

D

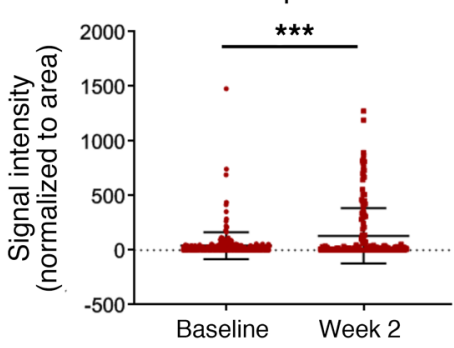

G

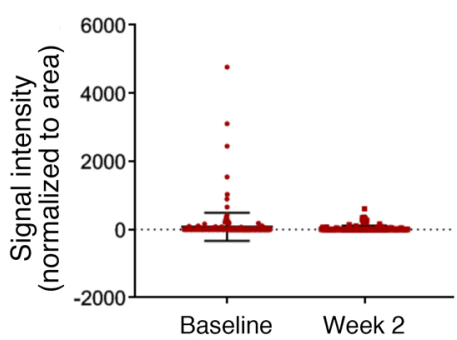

Figure 1. Multiplex IHC staining of tumor-draining lymph node biopsies from HNSCC patients before and after $\mathbf{2}$ weeks of CMT. (A) Representative images of tumor-draining lymph node biopsies from 15 HNSCC patients collected before and after 2 weeks of radiation therapy regimen. Original magnification, $\times 60$. Cyan, CD163+ M2 macrophages; green, p-STAT3; red, granulocytic CD15+ cells; yellow, CD8 ${ }^{+}$T cells; magenta, PD-L1; blue, DAPI (nuclei). (B-C) The indicated immune cell markers and p-STAT3 were stained on these sections using OPAL technology, and then imaged on the Vectra Automated Imaging System and quantified from the whole section. The signal intensities normalized to tissue area are shown for CD163+ M2 macrophages (B), p-STAT3 (C), colocalization of CD163 and p-STAT3 (D), granulocytic CD15+ cells (E), CD8 ${ }^{+}$T cells (F), and PD-L1 (C). Data presented as mean \pm SEM. ${ }^{*} P<0.05$, ${ }^{* * *} P<0.001$ by Student's $t$ test.

promotes MyD88/NF- $\kappa \mathrm{B}-$ dependent release of IL-6 by TAMs, thereby stimulating proangiogenic and tolerogenic STAT3 signaling and tumor relapse after RT (22). Our recent clinical study suggested that myeloid cell-dependent STAT3 signaling may also be limiting the efficacy of CMT in HNSCC patients (7). CMT-treated HNSCC patients showed elevated plasma levels of proinflammatory cytokines and chemokines together with the accumulation of immature myeloid cells with highly activated STAT3 in the circulation (7). Here, we verified these earlier observations using gene expression profiling and multiplexed immunostaining on HNSCC patient-derived tumor specimens collected before and 2 weeks into a 6- to 7-week course of RT. In addition, we have extended these studies to xenotransplanted and syngeneic mouse models of $\mathrm{HPV}^{+}$and $\mathrm{HPV}^{-}$head and neck tumors to evaluate the potential of using myeloid cell-targeted STAT3 antisense oligonucleotide (CpG-STAT3ASO) to reprogram the TME and to boost the immunogenic effects of RT.

\section{Results}

Accumulation of TAMs with activated STAT3 in lymph node biopsies from CMT-treated HNSCC patients. To assess the effect of CMT on the TME in HNSCC, we collected 15 fine-needle lymph node biopsies from patients before (baseline) and 2 weeks after treatment initiation. Various immune markers (CD163, CD8, and CD15) and immune checkpoint regulatory molecules (p-STAT3 and the PD-1 ligand, PD-L1) were stained and evaluated using fluorescence microscopy and digital image quantification (Figure 1A). RT induced significant recruitment of CD163+ TAMs (Figure 1B) and generally increased STAT3 activity (Figure 1C), which occurred mainly in TAMs (Figure 1D). In blood samples from the same group of HNSCC patients, we previously reported accumulation of circulating polymorphonuclear MDCSs (PMN-MDSCs) (HLA-DR CD14-CD15 ${ }^{+}$CD $33^{+}$; ref. 7). However, the total percentage of $\mathrm{CD}^{+} 5^{+}$cells in baseline tumor specimens was very low and decreased further after treatment (Figure 1E). 
A
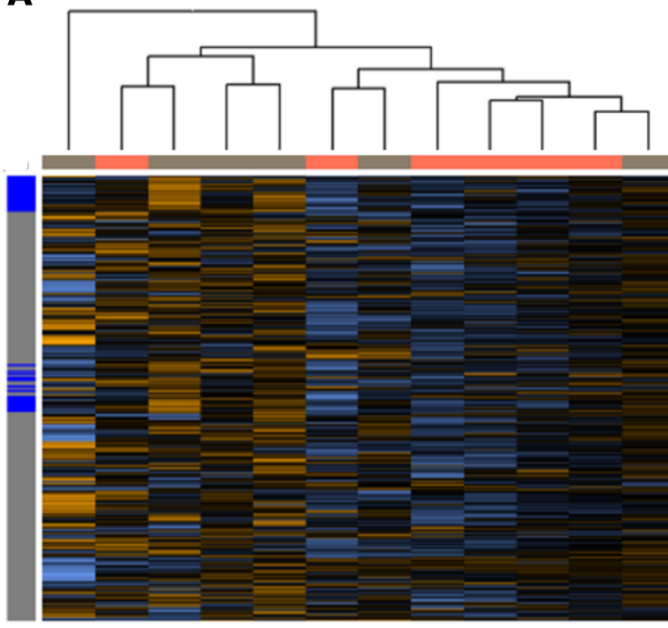

Baseline

Week 2

Probe annotations

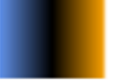

$-3-2-10123$ $z$ scores
B

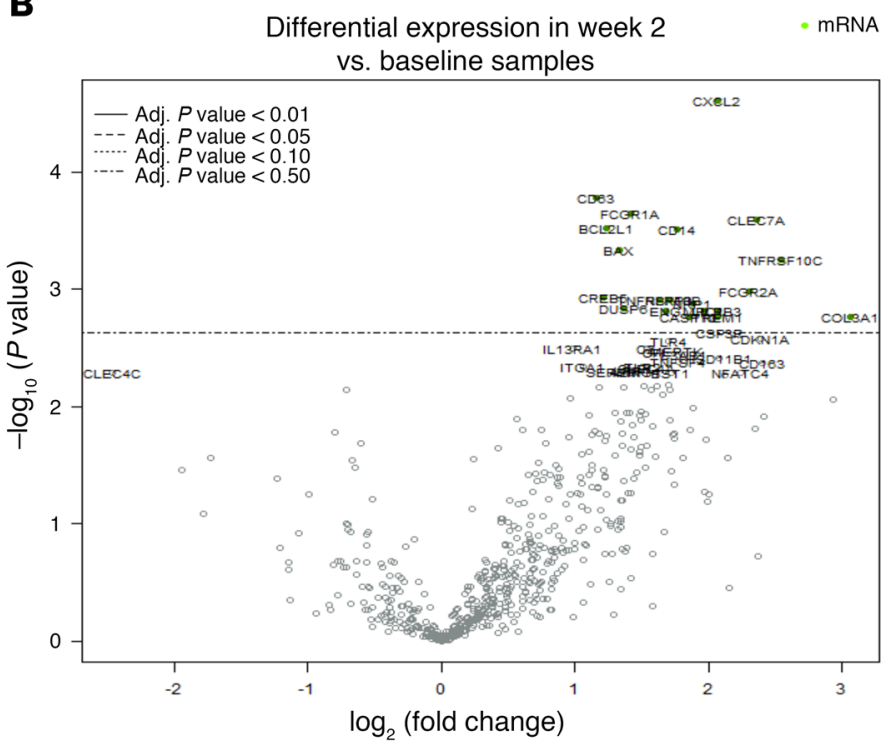

C

Macrophages vs. TILs

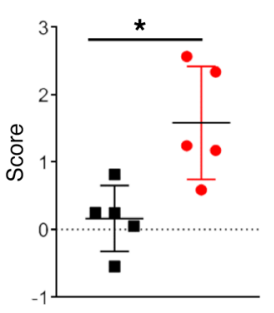

- Baseline - Week 2

E

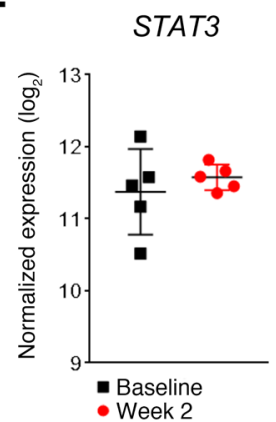

F

Cytotoxic cells vs. TILs

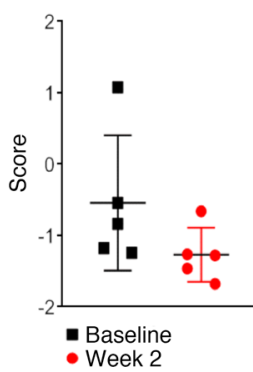

D

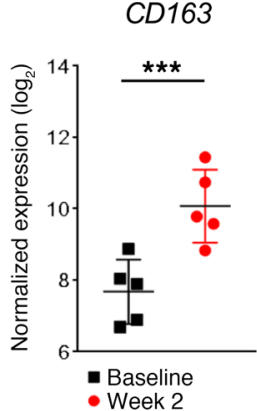

ARG1

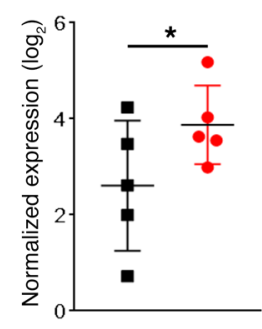

CTLA4

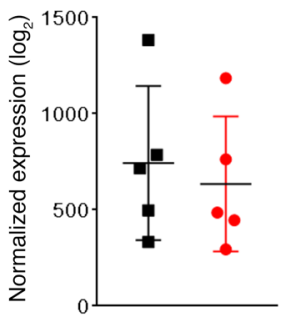

CD206

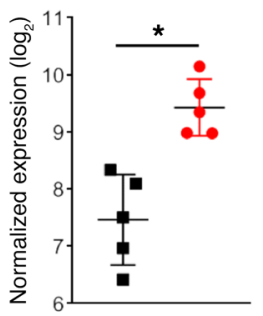

IL6

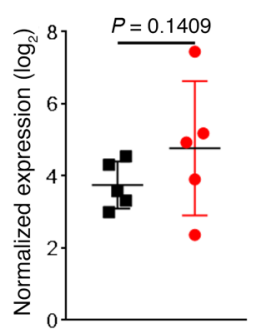

IDO

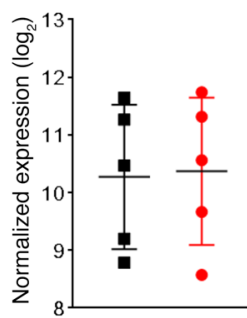

IL4
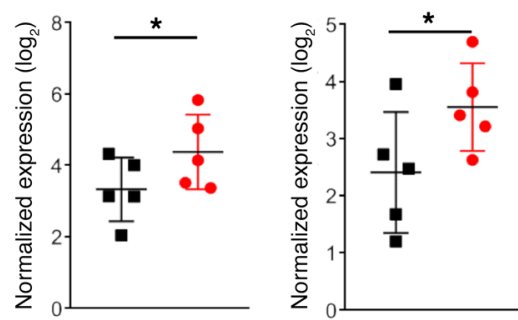

IL5

TGFB1

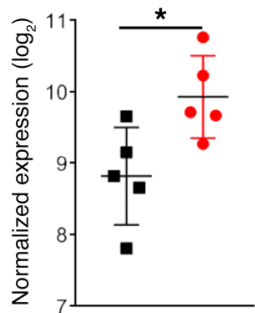

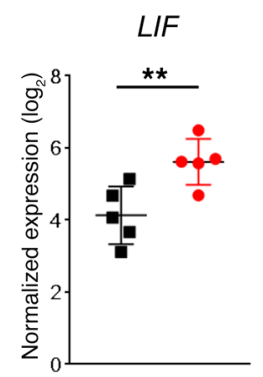

CD274/PD1L1

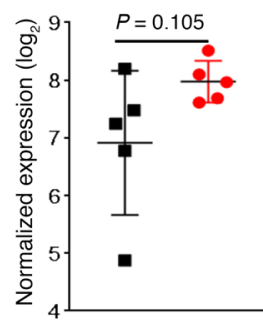

LAG3

PD1
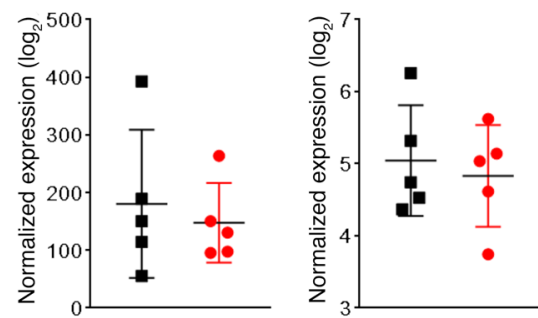
Figure 2. Changes in immune-related gene expression in the tumordraining lymph node biopsies from HNSCC patients after initial 2-week radiation therapy. Fine-needle biopsies were obtained from 6 HNSCC patients before and after radiation therapy and flash-frozen. The mRNA samples were analyzed using NanoString assay (Human Pancancer Immune panel) followed by data analysis in the nSolver software. (A) Clustering heatmap based on the similarity of the total changes in gene expression. (B) The average fold changes $\left(\log _{2}\right)$ in the 770 genes related to immune response are presented using a volcano plot. (C) Score for macrophage vs. total tumor-infiltrated lymphocytes (TILs) generated using advanced analysis in nSolver. (D) Expression of CD163 and CD206 and M2-related cytokines IL4, IL5, and TCFB1 in the tumor-containing Iymph node biopsies after CMT. (E) Expression of immune-suppressor STAT3-related genes at baseline and 2 weeks after CMT. Shown are the expression of STAT3, IL6, LIF, ARC1, and PDL1. (F) Lack of significant changes in scores for cytotoxic cells vs. TILs and expression of immune checkpoint regulators CTLA4, IDO, LAC3, and PD1 after radiotherapy. Data in $\mathbf{C}-\mathbf{F}$ represent the mean \pm SEM $(n=4) .{ }^{*} P<0.05$, ${ }^{* *} P<0.01,{ }^{* * *} P<0.001$ by Student's $t$ test.

This discrepancy may be related to differentiation of circulating MDSCs toward macrophages in the HNSCC TME, as suggested by others (23). In addition, we found a significant decrease in the percentage of $\mathrm{CD}^{+} \mathrm{T}$ cells after CMT (Figure 1F), while the low basal expression of immune checkpoint regulator PD-L1 in HNSCC specimens did not significantly change (Figure 1G). Altogether, these immunophenotyping results suggested that the initial stages of RT support expansion of TAMs and a STAT3driven tolerogenic microenvironment, which can potentially hinder long-term therapeutic effects.

$R T$ enhances tolerogenic gene signature in the head and neck $T M E$. To further characterize radiation-induced changes in the TME, we evaluated expression of 770 immune-related genes using NanoString assay and nCounter analysis. In the majority of patients, clustering analysis uncovered an increase in the overall gene expression after RT (Figure 2A). The major functional gene families increased as a result of the treatment were related to macrophage functions, senescence, and the TNF gene superfamily (Supplemental Figure 1; supplemental material available online with this article; https://doi.org/10.1172/JCI137001DS1). Thirty-six genes, including key markers of macrophage functions such as CD163, CSF3R, CXCL2, MSR1, and TREM1 showed differential expression after 2 weeks of CMT compared with baseline $(P<0.001$; Figure 2B and Supplemental Table 1$)$. Cell type profiling indicated significantly increased macrophage score versus tumor-infiltrating lymphocytes (TILs) $(P=0.0139)$ without a significant increase in neutrophil or total TIL score (Figure 2C and Supplemental Figure 1). Furthermore, we found significantly elevated expression of TAM markers CD163 $(P=0.0019)$ and CD206 $(P=0.0175)$ together with related M2 cytokines such as IL4 $(P=0.0359)$, IL5 $(P=0.0171)$, and TGFB1 $(P=0.0140)$ (Figure 2D). These data agree well with the increased accumulation of $\mathrm{CD}_{163}{ }^{+}$p-STAT3 ${ }^{+} \mathrm{M} 2$ macrophages after CMT, as revealed earlier by the immunofluorescent staining (Figure 1B). Although the STAT3 expression remained unaltered by the RT, there was a clear indication of STAT3 activity as suggested by increased expression in STAT3-regulated genes such as AGR1 $(P=0.01), \operatorname{LIF}(P=$ $0.001)$, and a relatively weak increase in $P D L 1(P=0.105)$ and IL6 $(P=0.1409)$ (Figure 2E). As expected, there was no significant difference in the cytotoxic cell versus TIL score after RT (Figure
2F). Also, there were no significant changes in the immune checkpoint regulators detectable in tumor specimens, such as CTLA4, PD1, IDO, or LAG3 (Figure 2F). Thus, the gene expression profiling of the irradiated HNSCC tumors suggests the presence of a potently immunosuppressive microenvironment dominated by tolerogenic macrophages.

STAT3 inhibition combined with TLR9 triggering stimulates human $T$ cell activity and inhibits growth of xenotransplanted head and neck tumors in humanized mice. We recently generated a strategy for targeted delivery of STAT3 antisense oligonucleotides into TLR9-positive myeloid cells (CpG-STAT3ASO), such as TAMs and MDSCs, associated with the microenvironment of solid tumors. The bifunctional CpG-STAT3ASO effectively disabled tolerogenic effects of human and mouse myeloid cells in vitro and in prostate cancer models in mice (24). Thus, we evaluated whether CpG-STAT3ASO will have similar effects on the TLR9+ PMN-MDSCs, which accumulate in HNSCC patients' blood $(7,25)$. We assessed the effect of CpG-STAT3ASO compared to control CpG-scrambled oligonucleotide (CpG-scrON) or STAT3ASO alone on the activation of human $\mathrm{CD}^{+} \mathrm{T}$ cells in the presence of PMN-MDSCs. CD15 ${ }^{+}$PMN-MDSCs enriched from blood of patients with advanced HNSCC were preincubated for 3 days in the presence of $500 \mathrm{nM} \mathrm{CpG-STAT3ASO,} \mathrm{STAT3ASO,}$ or CpG-scrON. Viable PMN-MDSCs were then cocultured (3:1) with allogeneic $\mathrm{CD}^{+} \mathrm{T}$ cells and $\mathrm{CD} 3 / \mathrm{CD} 28$ costimulation for an additional 3 days. As indicated in Figure 3A, only the bifunctional CpG-STAT3ASO alleviated the immunosuppressive effect of PMN-MDSCs on $\mathrm{CD}^{+} \mathrm{T}$ cell proliferation. Despite comparable levels of STAT3 knockdown by CpG-STAT3ASO and STAT3ASO alone, STAT3ASO did not restore $\mathrm{CD}^{+} \mathrm{T}$ cell proliferation, whereas the stimulatory effect of $\mathrm{CpG}$-scrON was significant but minor (Figure 3, A and B).

To verify the immunostimulatory potential of STAT3 inhibition/TLR9 activation on human immune cells in vivo, we generated a xenotransplanted HNSCC model of UM-SCC1 tumors in mice with a humanized immune system. Immunodeficient/transgenic NSG-SGM3 mice were first engrafted with human CD34 ${ }^{+}$cells. After 8 weeks, mice showing successful reconstitution of a human immune system (Supplemental Figure 2A) were engrafted s.c. with UM-SCC1 cancer cells. When tumors were established $\left(\sim 100 \mathrm{~mm}^{3}\right.$ volume), mice were treated every other day using intratumoral injections of CpG-STAT3ASO, control oligonucleotide STAT3ASO or CpG-scrON (5 mg/kg), or vehicle alone. Despite comparable levels of STAT3 inhibition by both CpG-STAT3ASO and control STAT3ASO as assessed using immunohistochemistry on tumor sections (Supplemental Figure 3A), only CpG-STAT3ASO, but not control treatments, arrested growth of UM-SCC1 xenotransplants (Figure 3C). These antitumor effects were likely immune mediated since CpG-STAT3ASO only weakly reduced the growth of the same tumor model in aged-matched immunodeficient NSG-SGM3 mice (Figure 3D). In fact, the immunophenotypic analysis of tumors collected from the humanized mice indicated that CpG-STAT3ASO induced the strongest activation of both myeloid immune cells and $\mathrm{T}$ cells compared with control treatments. Specifically, we observed a greater than 2-fold increase in the percentage of mature DCs (Figure 3E) and macrophages (Figure $3 \mathrm{~F}$ ) following CpG-STAT3ASO treatment, with a significant 
A
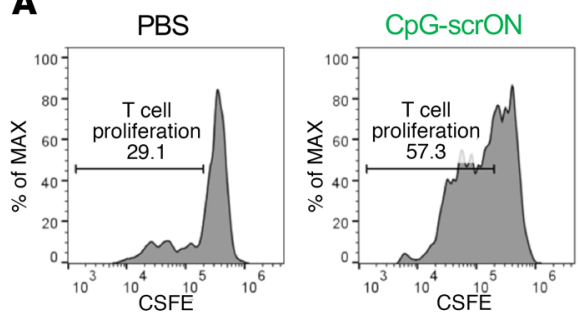

C Humanized mic

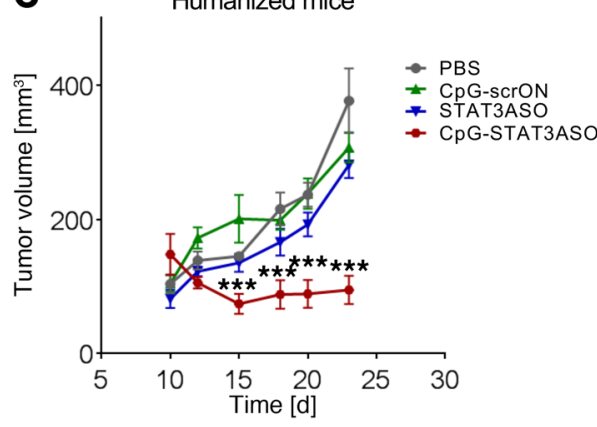

E
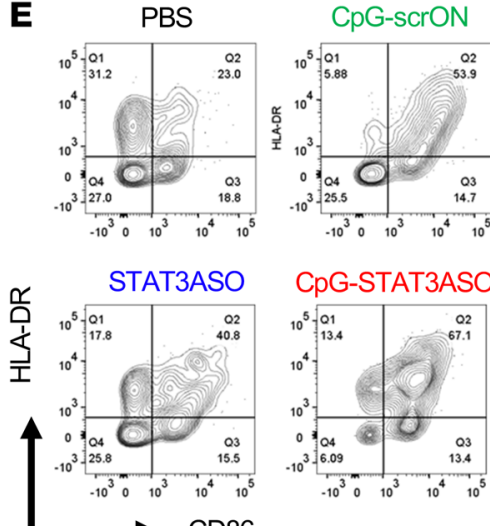

CD86
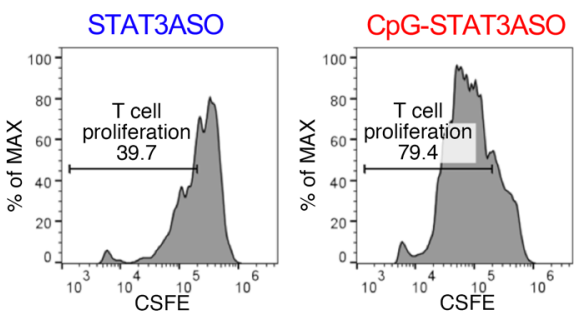

D Immunodeficient mice

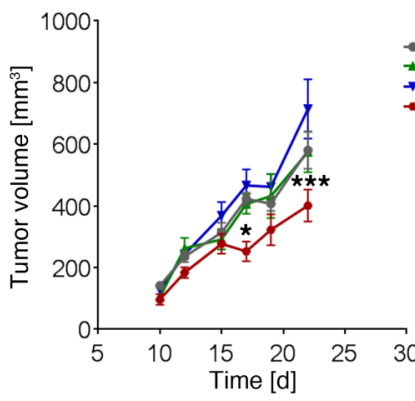

$\mathbf{F}$

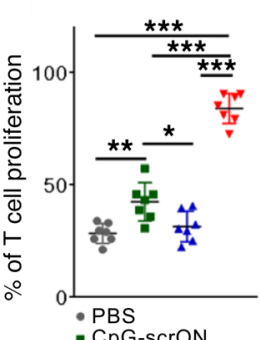

- CpG-scrON

- Cpg-STAT3ASO

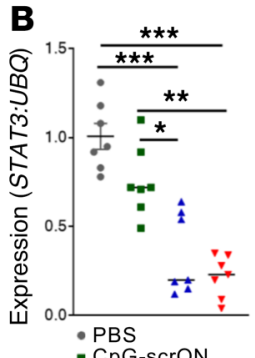

- CpG-scron : SPGATSATATZASO

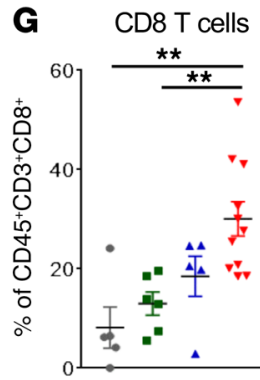

H

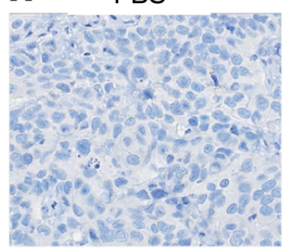

STAT3ASO

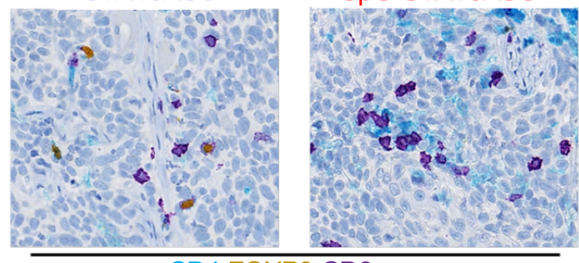

CD4 FOXP3 CD8

CD4 T cells

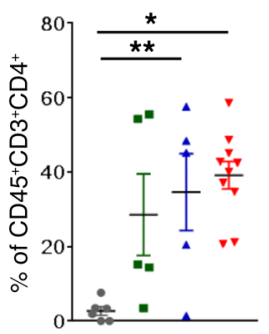

CpG-scrON

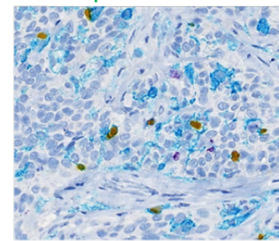

$$
\text { CpG-STAT3ASO }
$$

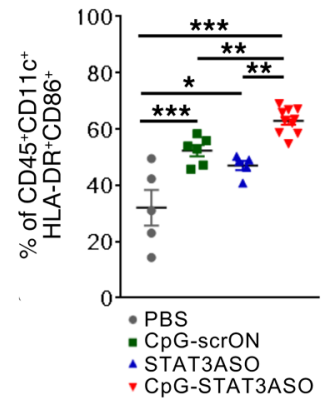

HLA-DR+CD86 ${ }^{+}$
PBS
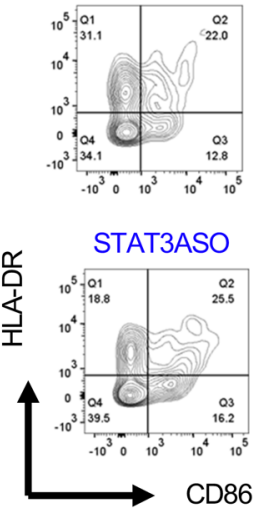

CD8: Treg ratio
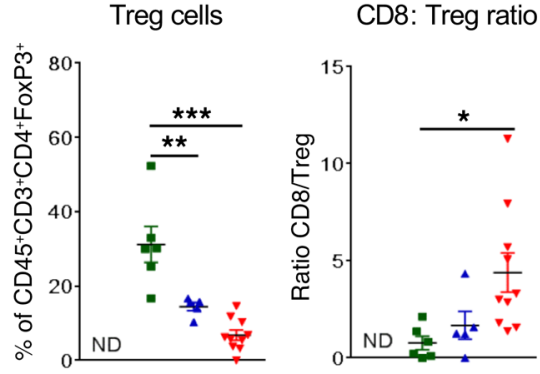

- PBS

- CPG-srcON

- Cpg-Stat3aso

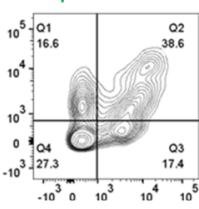

Cpg-STATZASO
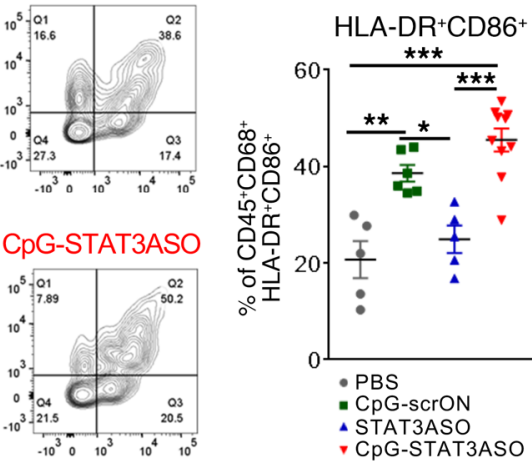
Figure 3. CpG-STAT3ASO stimulates human T cell activity and inhibits growth of xenotransplanted head and neck tumors in humanized mice. (A) $\mathrm{CD}_{15}{ }^{+}$granulocytic myeloid cells were enriched from PBMCs of HNSCC patients. Cells were treated using CpG-STAT3ASO, STAT3ASO alone, or control CpG-scrON $(0.5 \mu \mathrm{M})$ for 72 hours and then cocultured with allogeneic $\mathrm{CD}^{+} \mathrm{T}$ cells at a 3:1 ratio with anti-CD3/anti-CD28 costimulation. T cell proliferation was determined using CFSE dilution assay and flow cytometry $(n=7)$. (B) STAT3 knockdown in HNSCC CD15+ PMN-MDSCs after treatment using CpG-STAT3ASO, STAT3ASO alone, or control CpG-scrON $(0.5 \mu \mathrm{M})$ for 24 hours. Shown is STAT3 expression as measured using real-time qPCR $(n=7)$. UBQ, ubiquitin. (C-G) Immunodeficient NSG-SGM3 mice were humanized using i.v. injection of human $\mathrm{CD}^{+} 4^{+}$cells (C) or left untreated for control experiments (D). After 6 weeks, successfully humanized mice were injected s.c. with UM-SCC1 cells. Established UM-SCC1 tumors (150-200 $\left.\mathrm{mm}^{3}\right)$ were intratumorally treated with $5 \mathrm{mg} / \mathrm{kg}$ CpG-STAT3ASO $(n=10)$, CpG-scrON $(n=6)$, STAT3ASO $(n=6)$, or PBS $(n=6)$ every other day. $\operatorname{In}$ C and $\mathbf{D}$, tumor growth was measured every other day for the duration of the experiment. At the experiment completion, tumors were collected to assess immune cell infiltration using flow cytometry. (E) Percentage of activated dendritic cells (CD33+CD11 $\left.{ }^{+} \mathrm{HLA}-\mathrm{DR}^{+} \mathrm{CD}^{+} 6^{+}\right)$and (F) M1 macrophages (CD33+CD68+HLA-DR+CD86 ${ }^{+}$) in UM-SSC1 tumors after treatments. (C) Percentages of human T cells infiltrating the tumor after treatment. Shown are percentage of total $\mathrm{T}\left(\mathrm{CD} 5^{+} \mathrm{CD}^{+}\right), \mathrm{CD}^{+} \mathrm{T}\left(\mathrm{CD3}^{+} \mathrm{CD} 4^{+} \mathrm{CD} 8^{-}\right), \mathrm{CD}^{+}$ $\mathrm{T}\left(\mathrm{CD}^{+}{ }^{+} \mathrm{CD} 4{ }^{-} \mathrm{CD} 8^{+}\right)$, Treg $\left(\mathrm{CD3}^{+}{ }^{+} \mathrm{CD} 4^{+} \mathrm{CD}^{-} \mathrm{FoxP3}^{+}\right)$, and $\mathrm{CD} 8^{+} \mathrm{T} /$ Treg cell ratio as measured with flow cytometry $(n=6-10)$. (H) Representative images from multiplex immunohistochemical staining of UM-SCC1 tumor sections for $\mathrm{CD}^{+}$and Treg $\left(\mathrm{CD}^{+} \mathrm{FoxP3}^{+}\right)$cells (left) and for activated $\mathrm{CD} 8^{+} \mathrm{T}$ cells together with granzyme B and PD-1 (right). Scale bar: $50 \mu \mathrm{m}$. Data presented as mean \pm SEM. ${ }^{*} P<0.05,{ }^{* *} P<0.01,{ }^{* * *} P<0.001$ by 1 -way ANOVA with Tukey's multiple-comparison post hoc test (B and $\mathbf{E}-\mathbf{G}$ ) or 2-way ANOVA with Tukey's multiple-comparison post hoc test (C and $\mathbf{D}$ ).

but less pronounced effect of CpG-scrON and minimal immunostimulatory activity of STAT3ASO alone. These changes corresponded to a dramatic increase in the recruitment of $\mathrm{CD}^{+} \mathrm{T}$ cells into tumors, which contained $\mathrm{CD}^{+}$and $\mathrm{CD} 4^{+} \mathrm{T}$ cell populations (Figure 3, G and H, and Supplemental Figure 3B). Interestingly, Tregs were undetectable in vehicle-treated tumors but their population was increased after $\mathrm{CpG}$-scrON immunostimulation and to a lesser extent by STAT3ASO injections (Figure 3, G and H). In contrast, Treg infiltration into CpG-STAT3ASO-treated tumors remained minimal and correlated with a significant increase in the $\mathrm{CD}^{+}$/Treg ratio (Figure 3G), together with increased staining for granzyme B but not PD-1 (Figure 3H and Supplemental Figure 3C), positive indication of an adaptive antitumor immune response (5).

Local administration of CpG-STAT3ASO augments the efficacy of RT against HNSCC tumors in mice regardless of the HPV status. The xenotransplant tumor models in humanized mice are not suitable for long-term efficacy studies. Thus, we used 3 syngeneic models of $\mathrm{HPV}^{-}$(MOC2, mEERLsh) and $\mathrm{HPV}^{+}$(mEERL) head and neck cancers for testing the effect of STAT3 targeting on the outcome of RT. As shown in Figure 4A, 4 intratumoral injections of the CpG-STAT3ASO conjugate ( $5 \mathrm{mg} / \mathrm{kg}$ every other day), but not the unconjugated STAT3ASO alone or CpG-scrON, significantly reduced MOC2 tumor volume. These results contrasted with a significant reduction in STAT3 expression in whole MOC2 tumors by both CpG-STAT3ASO and STAT3ASO (Figure 4B). This is likely a result of the more efficient internalization of the CpG-STAT3ASO conjugate by cancer cells and by the tumor-associated myeloid cells, as demonstrated previously (24). Next, we assessed whether preconditioning the MOC2 TME using CpG-STAT3ASO treat- ment would enhance the efficacy of a single 13-Gy radiation dose. The combination of RT with 6 repeated injections of $5 \mathrm{mg} /$ $\mathrm{kg}$ CpG-STAT3ASO induced regression of MOC2 tumors in the majority of mice (Figure $4 \mathrm{C}$ ) and extended median animal survival to over 70 days (Figure 4D), compared with weak effects of either treatment alone. The CpG-STAT3ASO alone significantly delayed MOC2 tumor growth for over 20 days in a majority of mice but the RT alone reduced MOC2 tumor growth only temporarily within 10 days after irradiation (Figure 4C). Notably, the combined RT/CpG-STAT3ASO treatment resulted in growth inhibition and/or complete regression of MOC2 tumors in the treated and also in the distant, not directly treated sites, which suggests potential abscopal, systemic antitumor immune responses (Figure 4C). Furthermore, we evaluated the efficacy of the combination of CpG-STAT3ASO with RT in a metastatic $\mathrm{HPV}^{+} \mathrm{HNSCC}$ model, derived from mouse tonsil epithelial cells expressing HPV16 E6/ E7 plus the $H$-Ras oncogene together with luciferase (mEERL) (26). Similarly to previous studies $(26,27)$, mEERL tumors grew rapidly and showed resistance to a single dose of radiation (Figure $4 \mathrm{E})$. The repeated injections of CpG-STAT3ASO controlled tumor growth over the time of treatment but the combination of RT with CpG-STAT3ASO maximized therapeutic efficacy, inducing complete regression of $\mathrm{MEERL}$ tumors in half of the treated mice (Figure 4E). These results suggested that CMT improved the antitumor efficacy in both MOC2 and mEERL head and neck tumor models despite their different HPV status. In fact, the expression of HPV E6 and E7 oncoproteins in mEERL cells seemed to affect radiation sensitivity in this tumor model. The MEERL tumors with stably silenced E6/E7 genes (mEERLsh; refs. 26, 27) showed an enhanced antitumor effect after tumor irradiation (Figure $4 \mathrm{~F}$ ). The combination of RT with CpG-STAT3ASO resulted in more consistent mEERLsh tumor growth control compared with either treatment alone, although we did not observe tumor regression, unlike in the E6/E7-expressing mEERL (Figure $4 \mathrm{~F}$ vs. Figure $4 \mathrm{E}$ ). Other studies suggest that HPV E6/E7 oncoproteins may have 2 contradictory effects on head and neck tumors (3). They can promote cancer cell-intrinsic radioresistance of HNSCC, while at the same time being a source of targetable tumor-specific neoantigens for adaptive antitumor immune responses triggered by STAT3 targeting and TLR9 stimulation.

The combined RT and CPG-STAT3ASO shifts the balance of tolerogenic to immunogenic macrophages in the head and neck TME. To evaluate changes induced by the combined CpG-STAT3A$\mathrm{SO} / \mathrm{RT}$ in the HNSCC microenvironment, we characterized myeloid cell populations infiltrating MOC2 tumors using flow cytometry. We found a significant reduction in the putative M2

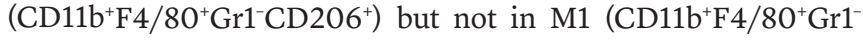
$\mathrm{CD}^{-}{ }^{-}$) macrophages only after the combined CpG-STAT3ASO/ RT treatment but not after either treatment alone $(P=0.0176$; Figure 5A and Supplemental Figure 4). Although the CMT had little effect on the percentage of tumor-associated MDSCs, it significantly reduced tolerogenic activity of STAT3 in this immunosuppressive population, similarly to that observed previously in patient-derived PMN-MDSCs (Figures 5B and 3A and Supplemental Figure 5) $(25,28)$. Next, we compared these observations to the changes in $\mathrm{HPV}^{+}$mEERL TME after similar treatments. As shown in Figure 5C, the combination of CpG-STAT3ASO/RT, 
A

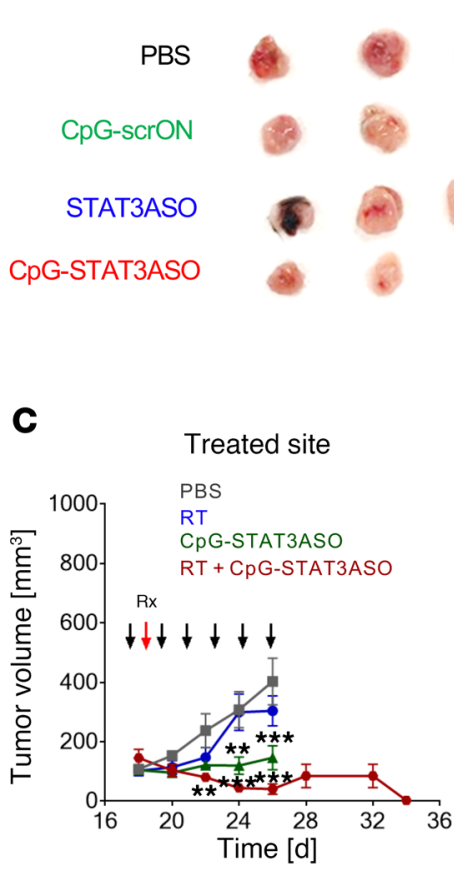

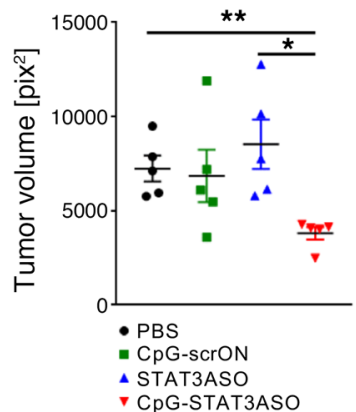

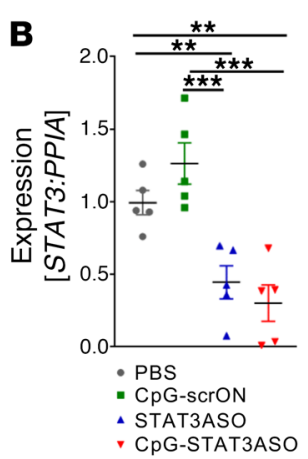

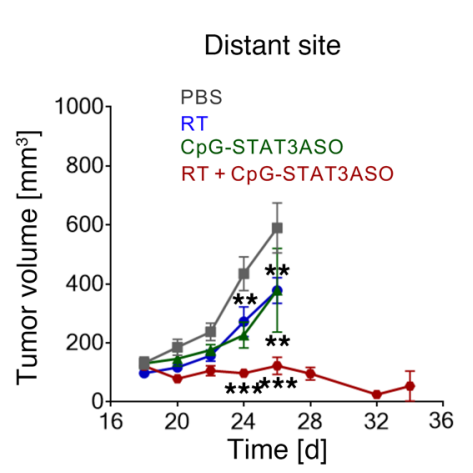

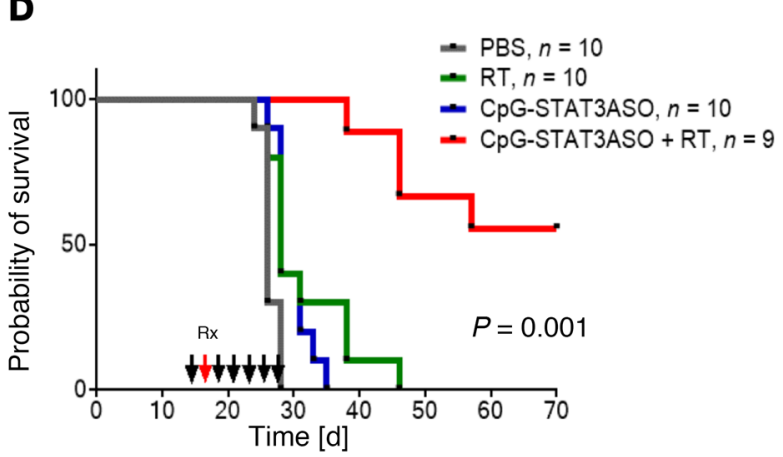

E

$\mathrm{HPV}^{+} \mathrm{mEERL}$
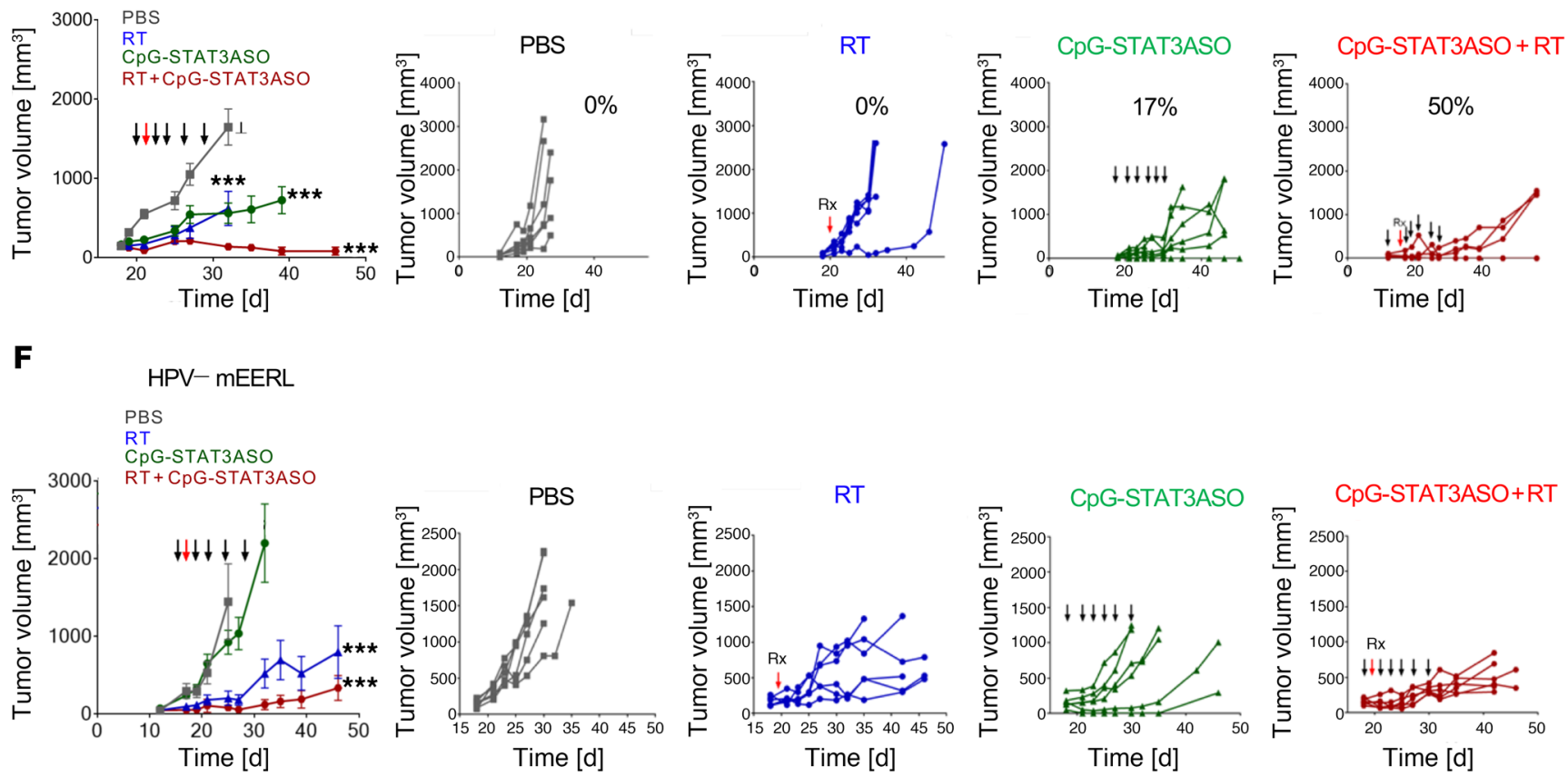

Figure 4. Local irradiation combined with intratumoral delivery of CpG-STAT3ASO improves the response to the irradiation in mice bearing HNSCC tumors independently of the HPV status. (A and B) C57BL/6 mice were injected s.c. in the flank with mouse syngeneic $\mathrm{HPV}^{+} \mathrm{mEERL}$. Established tumors were treated with $5 \mathrm{mg} / \mathrm{kg}$ CpG-STAT3ASO, STAT3ASO, CpG-srcON, or vehicle (PBS) every other day. After 4 treatments the tumors were collected, photographed, and weighed (A), and then STAT3 expression was measured using real-time qPCR (B) $(n=5)$. PPIA, peptidyl-prolyl cis-trans isomerase A. (C) HPVMOC2 cancer cells were engrafted in both flanks of mice to generate dual-tumor models. After tumors were established $\left(\sim 150 \mathrm{~mm}^{3}\right)$, mice were treated using CpG-STAT3ASO ( $5 \mathrm{mg} / \mathrm{kg}$ ) injected intratumorally every other day for a total of 6 times (black arrows), a single dose of local tumor irradiation (RT: $13 \mathrm{~Gy}$, red arrow), the combination of these treatments (CPG-STAT3ASO+RT), or vehicle alone (PBS). MOC2 tumor growth kinetics were measured using a caliper at treated (left) and at distant (right) tumor sites ( $n=6 /$ group). (D) The CpG-STAT3ASO plus RT combination treatment improves survival of MOC2 tumor-bearing mice; shown is the Kaplan-Meier survival curve $(n=9-10)$. (E and F) Comparison of antitumor effects of radiotherapy alone, CpG-STAT3A$\mathrm{SO}$ alone, and the combination of both. C57BL/6 mice were injected s.c. in the flank with syngeneic HPV+ $\mathrm{mEERL}$ (E) or HPV- $\mathrm{mEERL}^{-}$(F) HNSCC cells. Mice with established tumors were treated as described in C. Shown are representative results from 1 of 2 independent experiments ( $n=6 / g r o u p)$. Data presented as mean \pm SEM. ${ }^{*} P<0.05,{ }^{* *} P<0.01,{ }^{* *} P<0.001$ by 1-way ANOVA with Tukey's post hoc test (A and B) or 2-way ANOVA with Tukey's post hoc test (C, E, and $\mathbf{F})$. 
but not either treatment alone, reduced by half the percentage of tolerogenic CD206 ${ }^{+}$M2 TAMs while doubling the percentage of M1 macrophages. The CpG-STAT3ASO alone increased the percentage of M1 but had a weaker effect on M2 macrophages (Figure $5 \mathrm{C}$ ). A similar shift from the M2 to M1 phenotype was induced by the combined CpG-STAT3ASO/RT also in HPV $\mathrm{HERRLSh}^{-}$mE tumors (Figure 5D). In the absence of E6/E7 oncoproteins, RT alone also resulted in a moderate but significant effect, increasing the M1 to M2 macrophage ratio. Again, we did not observe significant changes in the percentage of infiltrating MDSCs in both $\mathrm{HPV}^{+}$ and $\mathrm{HPV}^{-}$control mEERL tumors (Supplemental Figure 5). These results suggest that the combination of CpG-STAT3ASO with RT can reprogram macrophage populations in the head and neck tumors, potentially jump starting antitumor immune responses.

Targeting TLR9/STAT3 in the head and neck TME promotes $R T$-induced accumulation of M1 macrophages in tumor-draining lymph nodes. The increased ratio of M1 to M2 macrophages in head and neck tumors correlated with the antitumor efficacy, suggesting that the combined CpG-STAT3ASO/RT can promote antigen presentation and adaptive $\mathrm{T}$ cell responses. Thus, we further assessed changes in the macrophage phenotype of MOC2 tumordraining lymph nodes (TDLNs) after CpG-STAT3ASO injection or CMT. In agreement with observations in the TME (Figure 5, A-D), we found increased percentages of mature, activated MHCI$\mathrm{I}^{+} \mathrm{CD} 80^{+}$and $\mathrm{MHCII}^{+} \mathrm{CD} 40^{+} \mathrm{M} 1$ macrophages in TDLNs from mice treated using the CpG-STAT3ASO/RT combination (Figure $5 \mathrm{E}$ and Supplemental Figure 6). CpG-STAT3ASO alone had a detectable but significantly weaker effect, while RT alone failed to induce macrophage activation. Similar results were derived from the $\mathrm{HPV}^{+} \mathrm{mEERL}$ tumor model, with the accumulation of activated M1 macrophages in TDLNs after the combined CpG-STAT3A$\mathrm{SO} / \mathrm{RT}$, although the effects were less obvious (Figure $5 \mathrm{~F}$ and Supplemental Figure 6). These findings correlate with the greater radioresistance of MEERL compared with MOC2 tumors (Figure 4). Interestingly, $\mathrm{HPV}^{-}$mEERLsh tumors lacking E6/E7 expression showed improved activation of the macrophage response to CpG-STAT3ASO/RT as well as CpG-STAT3ASO alone (Figure 5G and Supplemental Figure 5). Altogether, these results suggest that STAT3 inhibition/TLR9 stimulation induces migration of activated macrophages from the irradiated tumors to lymph nodes, thereby promoting antigen presentation and the adaptive antitumor immune responses.

$C D 8^{+}$and $C D 4^{+} T$ cells drive the antitumor immune response induced by the combination of RT with CPG-STAT3ASO. Our results so far strongly suggested the role of $\mathrm{T}$ cell-mediated immune responses in antitumor effects induced by the combined CpG-STAT3ASO/RT in selected HNSCC models. Thus, we used flow cytometry to characterize the effects of the tested therapeutic strategies on $\mathrm{T}$ cell populations in both tumors and TDLNs. As shown in Figure 6A, only the combined CpG-STAT3ASO/ RT treatment induced significant expansion of $\mathrm{CD}^{+} \mathrm{T}$ cells and increased the $\mathrm{CD}^{+} /$Treg ratio in TDLNs from MOC2 tumorbearing mice. The increased $\mathrm{CD} 8^{+} /$Treg ratio was also confirmed in MOC2 tumors; however, this effect was primarily driven by the reduced percentage of $\mathrm{CD} 4^{+} \mathrm{FoxP}^{+}$Tregs rather than $\mathrm{CD} 8^{+}$ $\mathrm{T}$ cell recruitment (Figure $6 \mathrm{~B}$ ). Nonetheless, these effects confirm the successful initiation of $\mathrm{T}$ cell immune responses against
MOC2 tumors by CpG-STAT3ASO/RT treatment. Similar effects were also observed in the $\mathrm{HPV}^{+} \mathrm{mEERL}$ tumor model. Again, the combined CpG-STAT3ASO/RT combination, but not RT or CpG-STAT3ASO alone, increased the $\mathrm{CD}^{+} /$Treg ratio in TDLNs (Figure 6C) as well as in tumors (Figure 6D). Finally, we assessed the contribution of $\mathrm{CD}^{+}$and $\mathrm{CD}^{+}{ }^{+} \mathrm{T}$ cells to CpG-STAT3ASO/ RT-induced antitumor immune responses in MOC2 tumor-bearing mice using an antibody-mediated depletion of each $\mathrm{T}$ cell population. As shown in Figure 6E, the depletion of either $\mathrm{CD}^{+}$ or $\mathrm{CD} 4^{+} \mathrm{T}$ cells completely abrogated the antitumor effect of the CpG-STAT3ASO/RT combination on MOC2 tumor progression and mouse survival (Figure 6E). Overall, our findings suggest that the immunogenic STAT3 inhibitor (CpG-STAT3ASO) can effectively reprogram tolerogenic myeloid cells in the TME, thereby sensitizing them to immunogenic effects of radiation and thereby facilitating effective $\mathrm{T}$ cell-mediated antitumor immunity against head and neck cancers independently of their HPV status.

\section{Discussion}

Successful induction of systemic antitumor immune responses is key to the long-term efficacy of cancer therapies, including RT $(12,21)$. Previous studies suggested that various types of cellular immune responses are associated with improved HNSCC patient survival. The recent immunogenomic classification of human cancers placed HNSCC in a group of IFN- $\gamma$-dominant tumors, characterized by strong M1/M2 macrophage polarization and a detectable CD8 signal (29). Correspondingly, the positive prognostic immune markers include the high infiltration by $\mathrm{CD}^{+} \mathrm{T}$ cells, lower percentage of circulating or tumor-infiltrating Tregs in $\mathrm{HPV}^{-}$ cancers, as well as an increased M1/M2 macrophage ratio (3, 30, 31). However, the underlying molecular mechanisms regulating these immune phenotypes or their contribution to RT resistance remain mostly undefined. Here, we provide evidence suggesting that tolerogenic STAT3 activity in macrophages infiltrating head and neck tumors provides a molecular mechanism restricting the efficacy of RT, not only against tumor models in mice, but also in HNSCC patients. The gene expression and microscopic analysis of biospecimens collected from $\mathrm{HPV}^{+} \mathrm{HNSCC}$ patients confirmed that clinical RT does not eliminate but rather favors expansion of immunosuppressive $\mathrm{CD} 163^{+} \mathrm{p}$-STAT3 ${ }^{+} \mathrm{M} 2$ macrophages in the TME with concurrent reduction in $\mathrm{CD}^{+} \mathrm{T}$ cell percentage. Our gene expression analysis confirmed that RT strengthens the immunosuppressive character of the HNSCC microenvironment, inducing a prominent STAT3-related gene signature, including $L I F$ and $A R G 1$, and decreasing markers of CTLs. Unlike in plasma samples as described in our earlier study (7) or in mouse tumor models, we did not find accumulation of potential PMN-MDSCs in HNSCC tumors. This discrepancy may reflect technical limitations in detecting human PMN-MDSCs or potential differentiation of MDSCs to macrophages after entering head and neck tumors, as observed by others (23). Previous studies demonstrated common expression of PD-1/PD-L1 and several other immune checkpoint regulators in head and neck tumors $(5,21)$. Although PD-L1 was detectable at both mRNA and protein levels in our analysis of patients' tumor specimens, it did not show significant upregulation after RT, similarly to other immune checkpoint molecules (PD-1, CTLA4, or LAG3). Nevertheless, head and neck tumors 
A
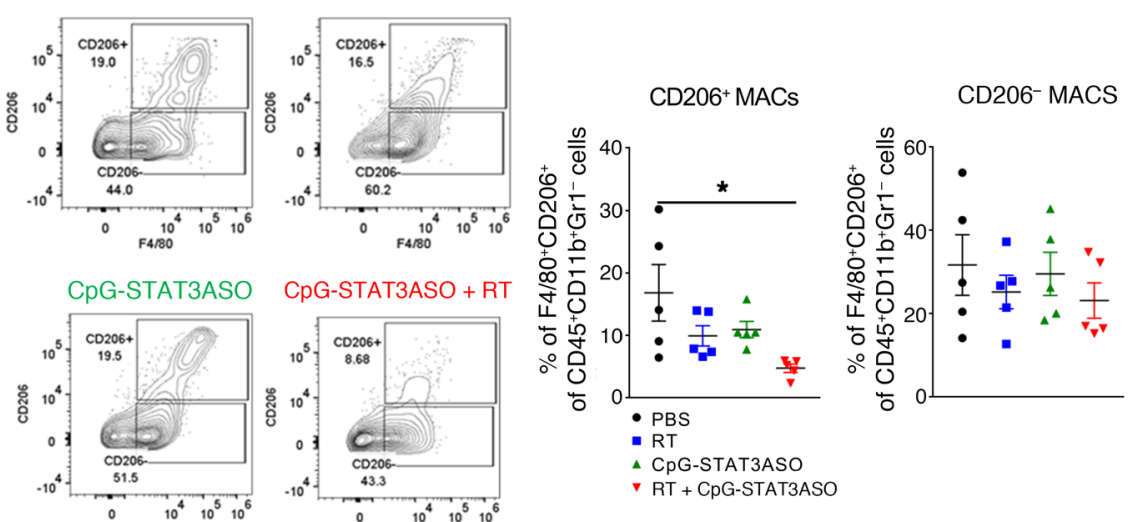

B

PMN-MDSC

PMN-MDSC/PSTAT3 ${ }^{+}$
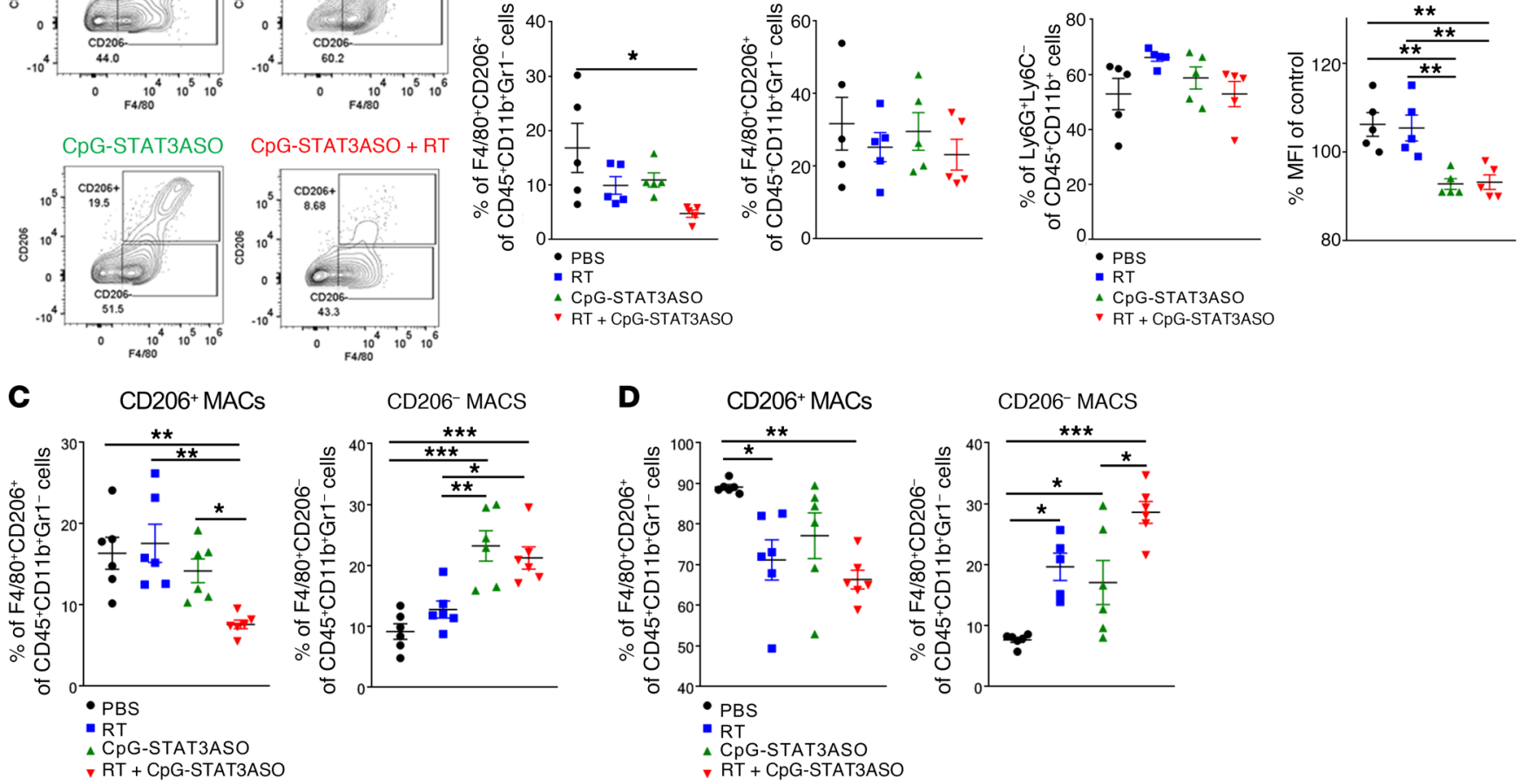

CD206- MACS

E

PBS

RT

CpG-STAT3ASO
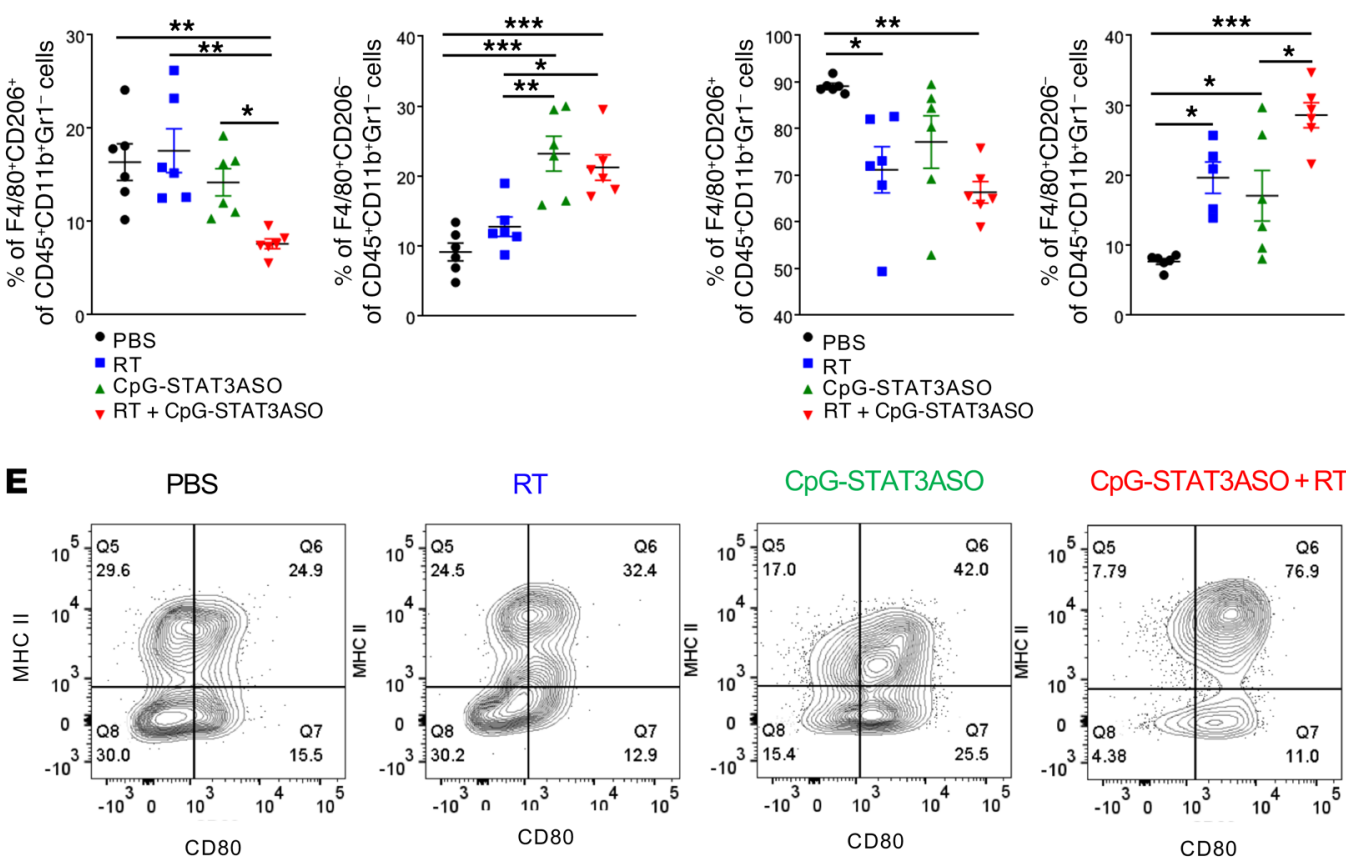

CpG-STAT3ASO + RT
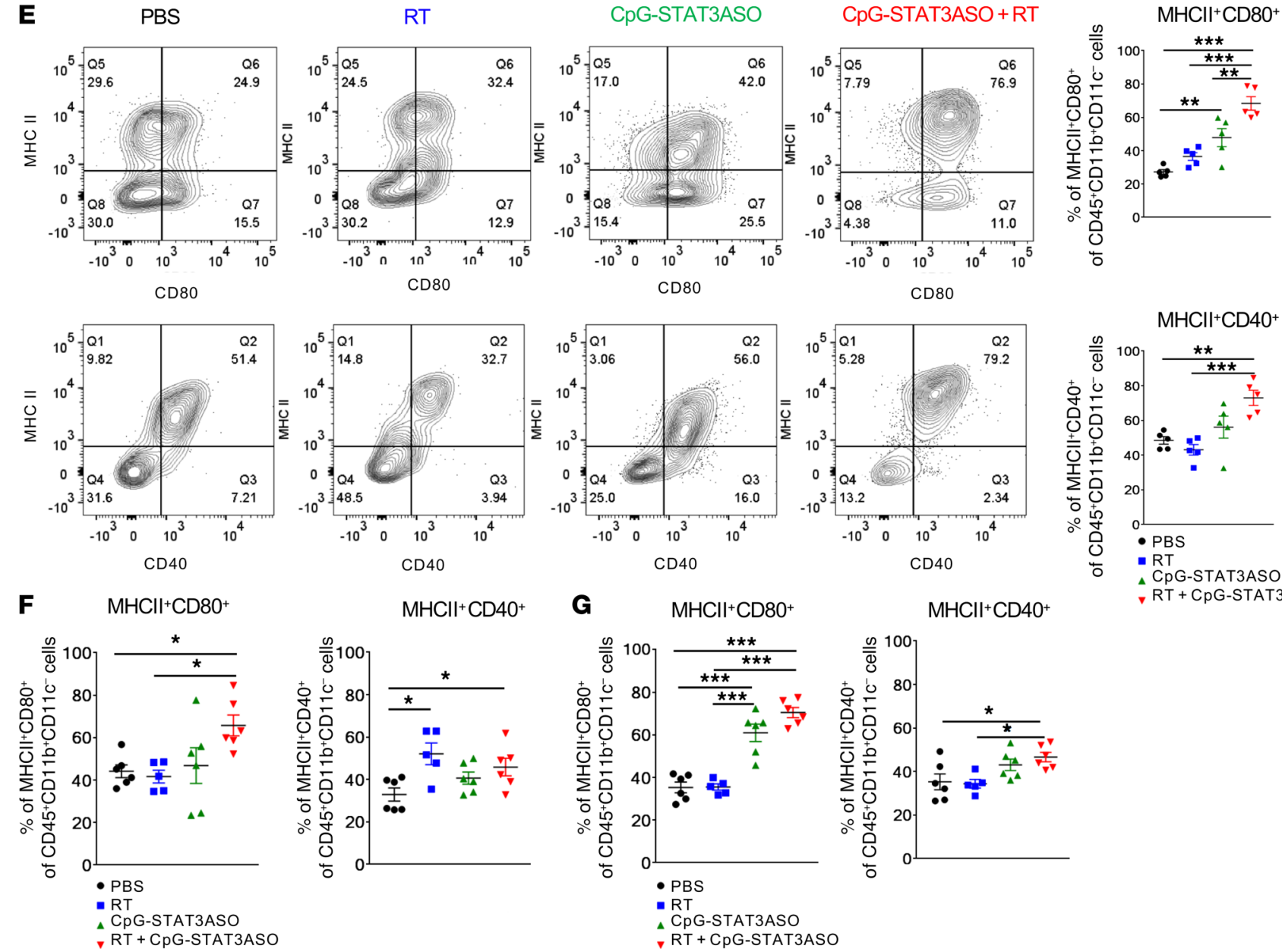

$\mathrm{MHCll}+\mathrm{CD} 40^{+}$
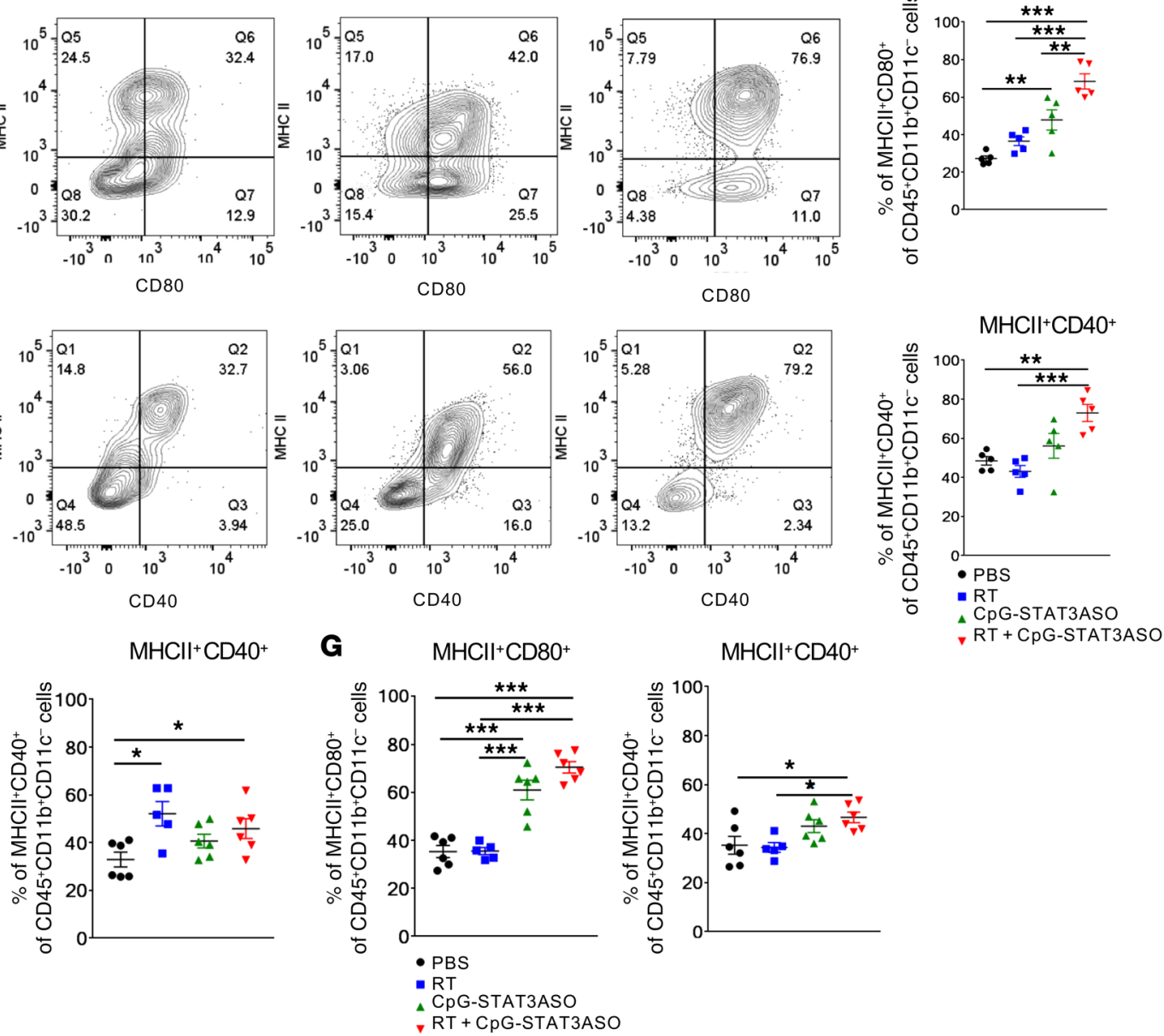
Figure 5. Combined therapy using CpG-STAT3ASO plus local tumor irradiation reduced the number of $\mathrm{M} 2$ macrophages in $\mathrm{HPV}^{-}$and $\mathrm{HPV}^{+}$tumors. $\mathrm{HPV}^{-} \mathrm{MOC2}, \mathrm{HPV}^{+} \mathrm{mEERL}$, or mEERLsh control tumors were established and treated as described in the Figure 4 legend. (A) Representative flow gating strategy and the percentages of $\mathrm{CD}^{206^{-}}\left(\mathrm{CD} 11 \mathrm{~b}^{+} \mathrm{CD} 206^{-} \mathrm{Cr} 1^{-} \mathrm{F} 4 / 80^{+}\right)$

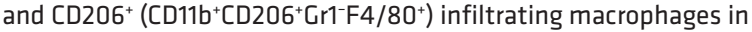
MOC2 tumors. Percentage of the infiltrating macrophages was analyzed using flow cytometry ( $n=6$ /group). (B) Percentages of PMN-MDSCs (CD11b+Ly6C ${ }^{+}{ }^{2} 6^{-10}{ }^{10}$ ) with p-STAT3 activation levels in HPV- MOC2 tumors analyzed using flow cytometry. (C and D) Percentages of CD206- and CD206 ${ }^{+}$infiltrating macrophages in $\mathrm{HPV}^{+}$mEERL (C) or $\mathrm{HPV}^{-}$mEERLsh (D) tumors as measured using flow cytometry. (E-G) The combination of CpG-STAT3ASO with tumor irradiation stimulates accumulation of activated macrophages in tumor-draining lymph nodes (after the initial 3 CpG-STAT3ASO injections) in MOC2 (E), mEERL (F), and mEERLsh tumorbearing mice (G). Data are representative results from 1 of 2 independent experiments and are presented as mean \pm SEM ( $n=6$ /group). ${ }^{*} P<0.05$, ${ }^{* *} P<0.01,{ }^{* *} P<0.001$ by 1 -way ANOVA with Tukey's post hoc test.

were positive for these immune checkpoint molecules, which agrees with recent preclinical and clinical studies demonstrating improved therapeutic effects of PD-1/PD-L1 blockade combined with TLR7 or TLR9 agonists $(32,33)$.

The increased M1/M2 macrophage ratio in $\mathrm{HPV}^{+}$compared with $\mathrm{HPV}^{-}$HNSCCs is a positive prognostic factor $(31,34)$ and a high percentage of M2 TAMs are associated with local and systemic immunosuppression in HNSCC (35). However, the molecular mechanisms driving tolerogenic macrophage activity in HNSCC are still elusive. Our observations in mouse models of HNSCC are consistent with findings in other solid tumors and the well-established role of STAT3 as a central regulator of tumor immune tolerance $(36,37)$. Importantly, as reported previously STAT3 can act as a negative feedback regulator and alter the outcome of tumor irradiation, shifting macrophage activity toward immunosuppression and angiogenesis (22). Our current study demonstrates that targeted inhibition of STAT3 combined with TLR9 triggering in HNSCC-associated macrophages using the CpG-STAT3ASO strategy overcomes radioresistance of both $\mathrm{HPV}^{+}$and $\mathrm{HPV}^{-}$tumors in mice. Therapeutic effects of the combined CpG-STAT3ASO/RT treatments correlated with a reduction in tumor-resident M2 macrophages and a concurrent recruitment of activated and mature M1 macrophages to TDLNs. These changes suggest disruption of the myeloid cell-driven immunosuppression in the local TME, also underscored by the expansion of $\mathrm{CD}^{+} \mathrm{T}$ cells and/or reduction of Treg percentages. Therefore, the local CpG-STAT3ASO/RT therapy can result in abscopal effects with the generation of long-term, systemic antitumor immune responses against distant HNSCC tumors. Oweida et al. have very recently suggested that STAT3 antisense oligonucleotides can enhance the efficacy of RT against HNSCC in mice through direct inhibition of STAT3 in $\mathrm{CD}^{+} \mathrm{T}$ cells, reducing Treg populations in tumors (38). However, due to poor penetration of antisense oligonucleotides into $\mathrm{T}$ cells in vivo $(24,39)$, the indirect, myeloid cell-dependent mechanism of T cell activation seems more likely. The results from the phase I clinical trial on STAT3ASO (AZD9150) in patients with various relapsed/ refractory tumors indicated that STAT3 was primarily targeted in tumor-associated myeloid cells, which were likely essential for the observed antitumor effects $(40,41)$. In addition, targeting STAT3 in T cell compartments is challenging, with often conflicting func- tional effects on diverse T cell subtypes. Although STAT3 reduces $\mathrm{CD}^{+} \mathrm{T}$ cell cytotoxicity (42) and enhances tolerogenic effects of Tregs (37), it is also critical for expansion of memory $\mathrm{T}$ cells (43) and long-term antitumor immunity (44). Our recent study compared the effect of STAT3ASO alone and the myeloid cellselective CpG-STAT3ASO against prostate cancer models in mice. We demonstrated that targeted delivery of STAT3ASO into myeloid cells together with CpG/TLR9 immunostimulation is necessary and sufficient for successful generation of antitumor immunity (24). Our current findings underscore the potential of using such myeloid cell-targeted STAT3 inhibition to break HNSCC resistance to $\mathrm{RT}$ and trigger $\mathrm{CD}^{+}$and $\mathrm{CD} 4^{+} \mathrm{T}$ cell-mediated antitumor immune responses. Both $\mathrm{CpG}$ oligodeoxynucleotides and STAT3ASO were well tolerated by patients, when tested as single agents in clinical trials $(45,46)$. The ongoing preclinical studies will assess safety and pharmacokinetic/pharmacodynamic properties of CpG-STAT3ASO. These results will serve as the foundation for a phase I clinical trial in patients with recurrent/metastatic HNSCC. The patients would be receiving RT in recurrent areas of gross disease concurrent with injections of CpG-STAT3ASO into a nodal tumor in the neck. We believe that this study can provide rationale for combining myeloid cell-selective inhibitors of STAT3 signaling with RT in order to augment the therapeutic efficacy and durability of responses in patients with not only HNSCC but also other solid tumors.

\section{Methods}

Clinical study design. Prospective biospecimens were collected from 15 HNSCC patients undergoing curative CMT. Patients had clinically palpable cervical nodes with elevated standard-uptake value (SUV) on a staging positron emission tomography-computed tomography (PET/CT). The eligible patients were diagnosed with a lymph nodepositive stage III/IV HNSCC (47) and received standard-of-care RT with concurrent cisplatin or cetuximab. Radiation $(66-70$ Gy total in 2- to 2.2-Gy fractions) was delivered using intensity-modulated RT. Fine-needle biopsies of tumor-containing lymph nodes (a maximum of 4 cores/patient) were performed by a board-certified radiologist, under ultrasound guidance, before and at 2 weeks into RT. Cores were immediately formalin-fixed or frozen. All biopsies were evaluated by a surgical pathologist for the presence of squamous cell carcinoma. Patient characteristics of the samples used in this study had been previously described (7).

Multiplex immunohistochemistry. The multiplexed analysis of immune markers was performed on the formalin-fixed, paraffinembedded fine-needle biopsies from 15 patients before (baseline) and after CMT. The sections were sequentially stained for CD15 (CM073A, Biocare Medical), CD8 (ab101500, Abcam), CD163 (ab201461, Abcam), PD-L1 (AC137A, Biocare Medical), and p-STAT3 (9131, Cell Signaling Technology) using the 7-color Opal kit (PerkinElmer). All slides were deparaffinized, rehydrated, and then boiled in antigen retrieval solution consisting of citrate buffer $(\mathrm{pH}$ 6.0) for the primary antibodies against PD-L1, CD15, CD163, and CD8, or in Tris-EDTA buffer $\mathrm{pH} 9.0$ for p-STAT3-specific antibody. The sections were blocked for 15 minutes using antibody diluent (Agilent Technologies), incubated for 30 minutes with primary antibodies at room temperature, and then incubated for 10 minutes with Opal Polymer HRP anti-mouse/rabbit IgGs before incubation for 10 minutes 
A

TDLN CD8

TDLN Treg

TDLN ratio CD8: Treg
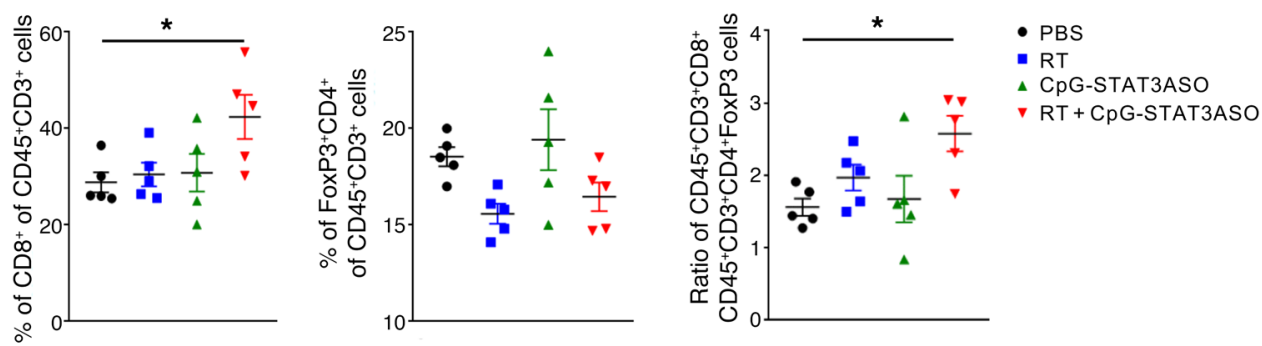

B
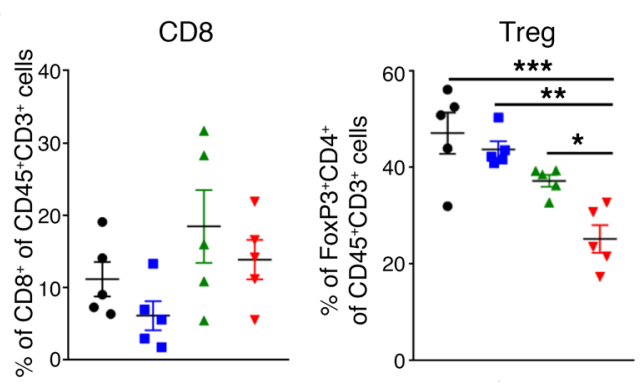

Ratio CD8: Treg
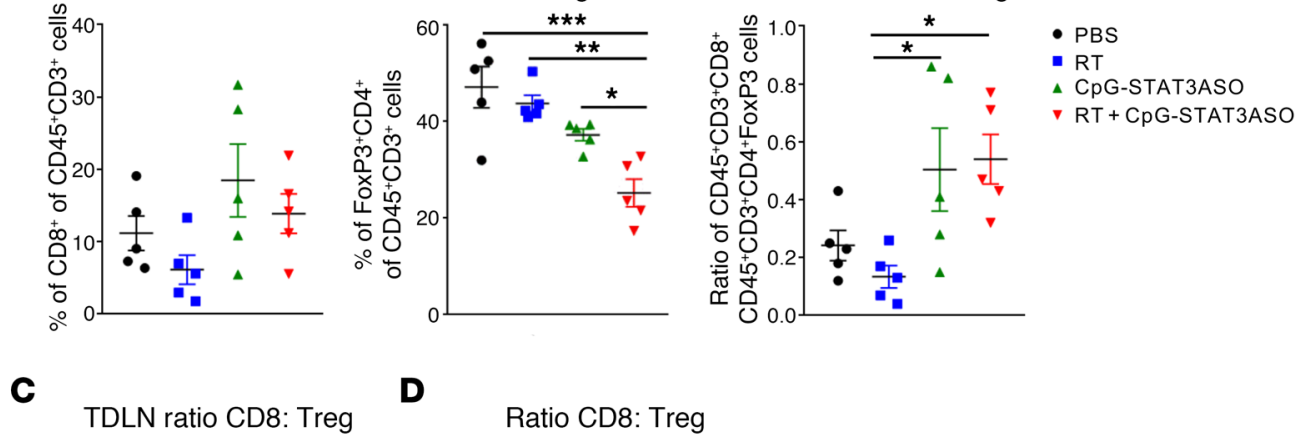

D

Ratio CD8: Treg
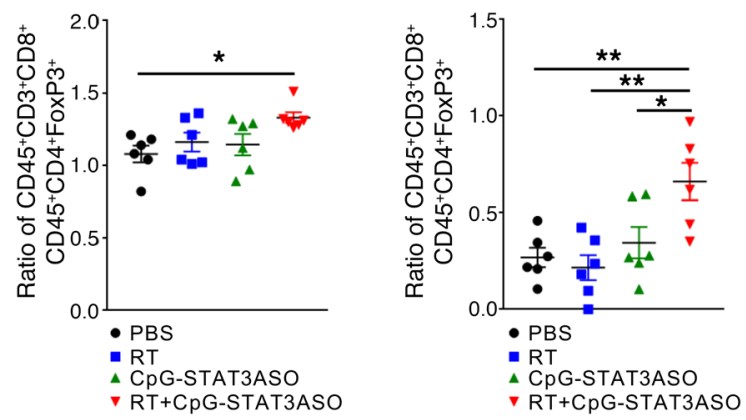

E
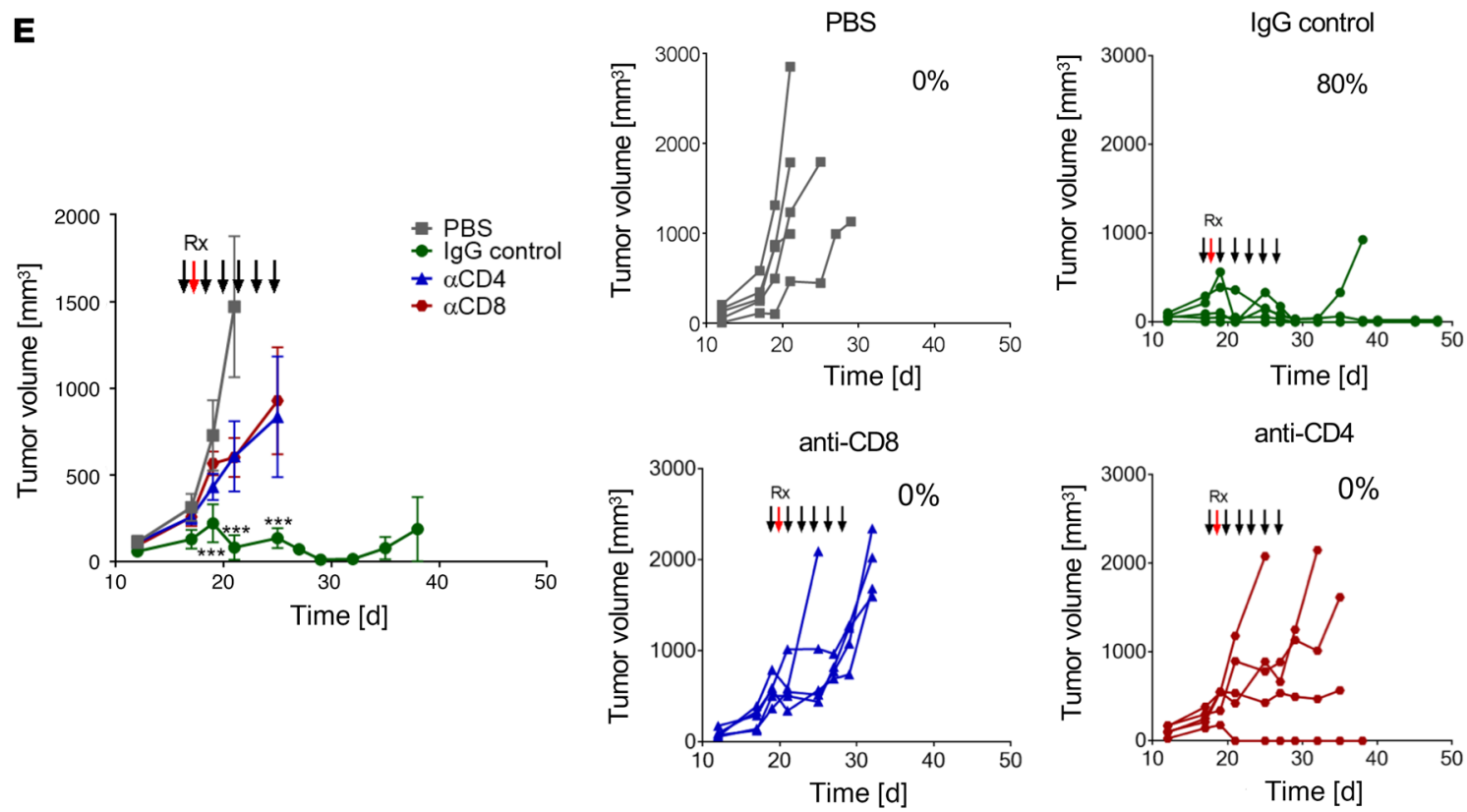
Figure 6. CD8 ${ }^{+} \mathrm{T}$ cell recruitment with concomitant decrease in regulatory $T$ cells drives the antitumor immune response induced by the combination of radiation therapy with CPG-STAT3ASO. MOC2 and $\mathrm{mEERL}$ tumors were established in immunocompetent mice and treated as described in the Figure 4 legend. Tumors and tumor-draining lymph nodes (TDLNs) were collected after the third treatment with CpG-STAT3ASO. (A and B) Percentages of CD8 ${ }^{+} \mathrm{T}$ cells and Tregs as well as the $\mathrm{CD}^{+} /$Treg ratio in TDLNs (A) or in MOC2 tumors (B) as measured by flow cytometry. (C and $\mathbf{D}$ ) The $\mathrm{CD8} 8^{+} /$Treg ratio in TDLNs (C) or in mEERL tumors (D). Shown are individual data points and mean \pm SEM ( $n=6$ /group). (E) CD8 ${ }^{+}$or CD4 ${ }^{+}$T cell depletion using specific antibodies abrogates the therapeutic antitumor effect of the combined CpG-STAT3ASO/radiotherapy against MOC2 tumors. After tumors were established $\left(\sim 150 \mathrm{~mm}^{3}\right)$, mice were treated using specific antibodies against CD4 and CD8 to deplete specific T cell subsets, control IgGs, or vehicle only (PBS). Subsequently, the mice were treated using local tumor irradiation (13 Gy) together with CpG-STAT3ASO ( $5 \mathrm{mg} / \mathrm{kg}$ intratumorally). The red arrow indicates tumor irradiation (Rx), black arrows indicate oligonucleotide injection. Data presented as mean $\pm \operatorname{SEM}(n=5 /$ group). ${ }^{*} P<0.05$, ${ }^{* *} P<0.01$, ${ }^{* *} P<0.001$ by 1 -way ANOVA with Tukey's post hoc test (A-D) or 2-way ANOVA with Tukey's post hoc test (E).

with the corresponding Opal 7-color fluorophores (Opal 520, 540, 620,650 , and 690). The slides were counterstained using DAPI and sealed in ProlongGold (Thermo Fisher Scientific). The whole sections were scanned and quantified with normalization to the tissue area on the Vectra system (PerkinElmer). Representative fields from the single-color slides were analyzed using InForm software v2.1 (PerkinElmer) to generate a spectral library. Index cases stained using the multiplex method were then imaged. Channels were unmixed using the spectral library, and the whole tissue sections were segmented, quantified, and normalized to the tissue area.

NanoString analysis. The total RNA from flash-frozen fine-needle biopsies obtained from 6 patients $\left(2 \mathrm{HPV}^{+}\right.$and $\left.4 \mathrm{HPV}^{-}\right)$before RT (baseline) and at 2 weeks after RT were extracted using a mirVANA kit (Thermo Fisher Scientific). The RNA quality was verified using the 2100 Bioanalyzer (Agilent). For gene expression analysis, we used the NanoString Human PanCancer Immune Profiling panel (XT-CSOHIP1-12) on the nCounter system (NanoString Technologies) following the manufacturer's recommendations. Data analysis was done using the included nSolver 4.0 software. Raw data were normalized using the stable housekeeping genes present in the panel. Heat-maps and cell profiling analysis were generated using the nSolver software v2.0.115.

Cell lines. $\mathrm{HPV}^{+}$oropharyngeal squamous cell carcinoma mEERL (mouse E6E7RasLuc) and genetically engineered $\mathrm{HPV}^{-}$control cells (mEERLshPTBLRas; mEERLsh) were described previously and provided by William Spanos (Sanford Research, Sioux Falls, South Dakota, USA) $(26,27)$. mEERL and mEERLsh were cultured in DMEM supplemented with 10\% FBS (Thermo Fisher Scientific), 1\% penicillin/ streptomycin (Thermo Fisher Scientific), EGF (10 ng/mL, Millipore), insulin $(10 \mathrm{mg} / \mathrm{mL})$, transferrin $(25 \mu \mathrm{g} / \mathrm{mL})$, cholera toxin $(0.25 \mathrm{~g} /$ $\mathrm{mL}$ ), and hydrocortisone $\left(25 \mathrm{~g} / \mathrm{mL}\right.$ ) (MilliporeSigma). HPV ${ }^{-}$murine oral cancer 2 (MOC2) cells were provided by Ravindra Upaluri (Dana-Farber Cancer Institute, Boston, Massachusetts, USA) and cultured in DMEM, 10\% FBS, 1\% penicillin/streptomycin, EGF (5 ng/ $\mathrm{mL}$ ), hydrocortisone (400 $\mathrm{ng} / \mathrm{mL})$, and insulin $(5 \mathrm{mg} / \mathrm{mL})$. UM-SCC1 (MiliporeSigma) were cultured in DMEM supplemented with $10 \%$ FBS and 1\% penicillin/streptomycin. All cells were tested bimonthly for mycoplasma contamination.
In vivo studies. Six- to 8 -week-old C57BL/6 mice were purchased from The Jackson Laboratory. For s.c. tumor growth experiments, 1.5 $\times 10^{5} \mathrm{mEERL}, \mathrm{mEERL}$, or MOC2 cells were injected s.c., and the tumor volumes were measured every other day. Tumors were considered established when they reached approximately $150 \mathrm{~mm}^{3}$ in size and locally irradiated (13 Gy) and/or injected intratumorally using 5 $\mathrm{mg} / \mathrm{kg}$ CpG-STAT3ASO or control CpG-scrON. For the humanized mouse model, 3- to 5-week-old NSG-SGM3 mice from The Jackson Laboratory were treated with whole body irradiation (120 cGy) 4 hours before i.v. injection of $1 \times 10^{5}$ human $\mathrm{CD} 34^{+}$hematopoietic stem cells, as previously described (48). After 6 weeks, fully humanized mice ( $\geq 25 \%$ human $\mathrm{CD} 45^{+}$cells in circulation) were implanted s.c. with UM-SCC1 HNSCC cells.

Flow cytometry. Flow cytometry was used for characterization of the TME on single-cell suspensions prepared by mechanical dispersion and enzymatic digestion of tumors and TDLNs. Extracellular staining was performed using fluorochrome-labeled antibodies against human HLA-DR (LN3), CD3 (OKT3), CD4 (OKT4), CD8 (RPA-T8), CD11c (3.9), CD45 (HI30), CD86 (IT2.2), FOXP3 (236A/E7) (all eBiosciences); or mouse major histocompatibility complex (MHC) class II (AF6120.1), CD3 (145-2C11), CD4 (RM4-5), CD8 (53-6.7), CD11b (M1/70), CD31 (clone 390), CD40 (HM40-3), CD45 (30-F11), CD68 (Y1/82A), CD80 (16-10A1), CD86 (GL1), CD206 (MR5D3), F4/80 (BM8), Ly6C (HK1.4), Ly6G (RB6-8C5) (all Thermo Fisher Scientific), and Ly6B.2 (ab53453, Abcam), after blocking with anti-Fc $\gamma$ III/IIR (2.4G2, Thermo Fisher Scientific). For intracellular staining, cells were fixed and permeabilized before immunostaining for p-STAT3 (9131, Cell Signaling Technology), FoxP3 (FJK-16S, Thermo Fisher Scientific), or arginase-1 (IC568A, R\&D Systems), as previously described (49). Fluorescence data were collected on the BD Fortessa cytometer and analyzed using FlowJo software (TreeStar).

Statistics. A paired Student's $t$ test was used to calculate 2-tailed $P$ values to estimate statistical significance of differences between 2 treatment groups. One- or 2-way ANOVA plus Tukey's multiple-comparison post hoc test was applied to assess differences between multiple groups or in the tumor growth kinetics experiments. Kaplan-Meier survival curve analysis with log-rank test was used to determine statistical significance in animal survival studies. Statistically significant $P$ values are indicated in figures as follows: ${ }^{*} P<0.05,{ }^{* *} P<0.01,{ }^{* *} P$ $<0.001$. Data were analyzed using Prism software v6.01 (GraphPad).

Study approval. The study protocol using clinical biospecimens including the relevant informed consent form was approved by the institutional review board at City of Hope (IRB14255). The study was conducted in accordance with the amended Declaration of Helsinki and the International Conference on Harmonization Guidelines. Mouse care and experimental procedures were performed under pathogen-free conditions in accordance with established institutional guidance and approved protocols from the City of Hope Institutional Animal Care and Use Committee.

\section{Author contributions}

SS and MK conceived and designed the study. DM, HW, SS, MA, and CW acquired data. DM, HW, SS, YLS, E Massarelli, and MK analyzed and interpreted the data. MK, SS, DM, and SVW wrote the manuscript. SS, E Maghami, E Massarelli, and PL provided technical and/or material support. MK, SS, and E Massarelli supervised the study. 


\section{Acknowledgments}

We would like to acknowledge the dedication of staff members at the City of Hope Analytical Cytometry, DNA/RNA Synthesis, Molecular Pathology, and Pathology Cores and the Animal Resources Center. Specifically, we are grateful to Holly Yin and Raju Pillai (Molecular Pathology) for technical assistance. We are grateful to William Spanos for kindly providing mEERL tumor models. We are also thankful to Chris McIntyre and the STOP CANCER Foundation for their generosity and partial funding of this project. This work was also partly supported by the National
Cancer Institute of the NIH under grants R01CA215183 (to MK) and P30CA033572 (to City of Hope). The content is solely the responsibility of the authors and does not necessarily represent the official views of the NIH.

Address correspondence to: Marcin Kortylewski, 1500 E Duarte Rd., City of Hope, Duarte, CA 91010, USA. Phone: 626.218.4120; Email: mkortylewski@coh.org. Or to: Erminia Massarelli, 1500 E Duarte Rd., City of Hope, Duarte, CA 91010, USA. Phone: 626.218.9200; Email: emassarelli@coh.org.
1. Ang KK, et al. Human papillomavirus and survival of patients with oropharyngeal cancer. $N$ Engl J Med. 2010;363(1):24-35.

2. Wagner S, et al. Human papillomavirus association is the most important predictor for surgically treated patients with oropharyngeal cancer. Br J Cancer. 2017;116(12):1604-1611.

3. Wang H-F, et al. The double-edged sword-how human papillomaviruses interact with immunity in head and neck cancer. Front Immunol. 2019;10:653.

4. Forster MD, Devlin MJ. Immune checkpoint inhibition in head and neck cancer. Front Oncol. 2018;8:310.

5. Mandal R, et al. The head and neck cancer immune landscape and its immunotherapeutic implications. JCI Insight. 2016;1(17):e89829.

6. Whiteside TL. Head and neck carcinoma immunotherapy: facts and hopes. Clin Cancer Res. 2018;24(1):6-13.

7. Sampath $\mathrm{S}$, et al. Combined modality radiation therapy promotes tolerogenic myeloid cell populations and STAT3-related gene expression in head and neck cancer patients. Oncotarget. 2018;9(13):11279-11290.

8. Fu KK. Combined-modality therapy for head and neck cancer. Oncology (Williston Park). 1997;11(12):1781-1790.

9. Leemans CR, et al. The molecular biology of head and neck cancer. Nat Rev Cancer. 2011;11(1):9-22.

10. Karar J, Maity A. Modulating the tumor microenvironment to increase radiation responsiveness. Cancer Biol Ther. 2009;8(21):1994-2001.

11. Garcia-Barros M, et al. Tumor response to radiotherapy regulated by endothelial cell apoptosis. Science. 2003;300(5622):1155-1159.

12. Formenti SC, Demaria S. Systemic effects of local radiotherapy. Lancet Oncol. 2009;10(7):718-726.

13. Noy R, Pollard JW. Tumor-associated macrophages: from mechanisms to therapy. Immunity. 2014;41(1):49-61.

14. Duray A, et al. Immune suppression in head and neck cancers: a review. Clin Dev Immunol. 2010;2010:701657.

15. Ahn G-O, et al. Inhibition of Mac-1 (CD11b/ CD18) enhances tumor response to radiation by reducing myeloid cell recruitment. Proc Natl Acad Sci U S A. 2010;107(18):8363-8368.

16. Barker HE, et al. The tumour microenvironment after radiotherapy: mechanisms of resistance and recurrence. Nat Rev Cancer. 2015;15(7):409-425.

17. Whiteside TL. Immunobiology of head and neck cancer. Cancer Metastasis Rev. 2005;24(1):95-105.
18. Apetoh L, et al. Toll-like receptor 4-dependent contribution of the immune system to anticancer chemotherapy and radiotherapy. Nat Med. 2007;13(9):1050-1059.

19. Pries R, et al. Toll-like receptor modulation in head and neck cancer. Crit Rev Immunol. 2008;28(3):201-213.

20. Rovere-Querini P, Castiglioni A. Adjuvant role for cell death during chemo - and radiotherapy of cancer? Expert Rev Clin Immunol. 2008;4(1):27-32.

21. Pilones KA, et al. Combination of radiotherapy and immune checkpoint inhibitors. Semin Radiat Oncol. 2015;25(1):28-33.

22. Gao C, et al. TLR9 signaling in the tumor microenvironment initiates cancer recurrence after radiotherapy. Cancer Res. 2013;73(24):7211-7221.

23. Corzo CA, et al. HIF- $1 \alpha$ regulates function and differentiation of myeloid-derived suppressor cells in the tumor microenvironment. JExp Med. 2010;207(11):2439-2453.

24. Moreira D, et al. STAT3 inhibition combined with CpG immunostimulation activates antitumor immunity to eradicate genetically distinct castration-resistant prostate cancers. Clin Cancer Res. 2018;24(23):5948-5962.

25. Vasquez-Dunddel D, et al. STAT3 regulates arginase-I in myeloid-derived suppressor cells from cancer patients. JClin Invest. 2013;123(4):1580-1589.

26. Hoover AC, et al. The role of human papillomavirus $16 \mathrm{E} 6$ in anchorage-independent and invasive growth of mouse tonsil epithelium. Arch Otolaryngol Head Neck Surg. 2007;133(5):495-502.

27. Vermeer DW, et al. Metastatic model of HPV oropharyngeal squamous cell carcinoma demonstrates heterogeneity in tumor metastasis. Oncotarget. 2016;7(17):24194-24207.

28. Won $\mathrm{H}$, et al. TLR9 expression and secretion of LIF by prostate cancer cells stimulates accumulation and activity of polymorphonuclear MDSCs. JLeukoc Biol. 2017;102(2):423-436.

29. Thorsson V, et al. The immune landscape of cancer. Immunity. 2018;48(4):812-830.e14.

30. Arce Vargas F, et al. Fc-optimized anti-CD25 depletes tumor-infiltrating regulatory $\mathrm{T}$ cells and synergizes with PD-1 blockade to eradicate established tumors. Immunity. 2017;46(4):577-586.

31. Kumar AT, et al. Prognostic significance of tumor-associated macrophage content in head and neck squamous cell carcinoma: a metaanalysis. Front. Oncol. 2019;9:656.

32. Sato-Kaneko F, et al. Combination immunotherapy with TLR agonists and checkpoint inhibitors suppresses head and neck cancer. JCI Insight. 2017;2(18):e93397.
33. Krieg AM. Development of TLR9 agonists for cancer therapy. J Clin Invest. 2007;117(5):1184-1194.

34. Chen $X$, et al. Immunological network analysis in HPV associated head and neck squamous cancer and implications for disease prognosis. Mol Immunol. 2018;96:28-36.

35. Costa NL, et al. Tumor-associated macrophages and the profile of inflammatory cytokines in oral squamous cell carcinoma. Oral Oncol. 2013;49(3):216-223.

36. Kortylewski M, et al. Inhibiting Stat3 signaling in the hematopoietic system elicits multicomponent antitumor immunity. Nat Med. 2005;11(12):1314-1321.

37. Kortylewski M, et al. Regulation of the IL-23 and IL-12 balance by Stat 3 signaling in the tumor microenvironment. Cancer Cell. 2009;15(2):114-123.

38. Oweida AJ, et al. STAT3 modulation of regulatory $\mathrm{T}$ cells in response to radiation therapy in head and neck cancer. J Natl Cancer Inst. 2019;111(12):1339-1349.

39. Crooke ST, et al. Cellular uptake and trafficking of antisense oligonucleotides. Nat Biotechnol. 2017;35(3):230-237.

40. Woessner RD, et al. Abstract A94: STAT3 antisense treatment decreases M2 macrophage infiltration and enhances the activity of checkpoint inhibitors in preclinical tumor models. $\mathrm{Mol}$ Cancer Ther. 2015;14(12 supplement 2):A94-A94.

41. Kroemer G, et al. STAT3 inhibition for cancer therapy: Cell-autonomous effects only? Oncoimmunology. 2016;5(5):e1126063.

42. Kujawski M, et al. Targeting STAT3 in adoptively transferred $\mathrm{T}$ cells promotes their in vivo expansion and antitumor effects. Cancer Res. 2010;70(23):9599-9610.

43. Kaech SM, Cui W. Transcriptional control of effector and memory $\mathrm{CD}^{+} \mathrm{T}$ cell differentiation. Nat Rev Immunol. 2012;12(11):749-761.

44. Huynh J, et al. Therapeutically exploiting STAT3 activity in cancer - using tissue repair as a road map. Nat Rev Cancer. 2019;19(2):82-96.

45. Krieg AM. CpG still rocks! Update on an accidental drug. Nucleic Acid Ther. 2012;22(2):77-89.

46. Hong D, et al. AZD9150, a next-generation antisense oligonucleotide inhibitor of STAT3 with early evidence of clinical activity in lymphoma and lung cancer. Sci Transl Med. 2015;7(314):314ra185.

47. Edge SB, Compton CC. The American Joint Committee on Cancer: the 7th edition of the AJCC cancer staging manual and the future of TNM. Ann Surg Oncol. 2010;17(6):1471-1474. 
48. Xia X, et al. Humanized NOD/SCID/ IL2rynull (hu-NSG) mouse model for HIV replication and latency studies. J Vis Exp.
2019;(143):10.3791/58255.

49. Hossain DMS, et al. TLR9-targeted STAT3 silencing sbrogates immunosuppressive activity of myeloid-derived suppressor cells from prostate cancer patients. Clin Cancer Res. 2015;21(16):3771-3782. 